\title{
Apalutamide Absorption, Metabolism, and Excretion in Healthy Men, and Enzyme Reaction in Human Hepatocytes ${ }^{\mathbb{S}}$
}

\author{
Ronald de Vries, Frank Jacobs, Geert Mannens, Jan Snoeys, Filip Cuyckens, \\ Caly Chien, and Peter Ward
}

\begin{abstract}
Janssen Research \& Development, Beerse, Belgium (R.d.V., F.J., G.M., J.S., F.C.); Janssen Research \& Development, Spring House, Pennsylvania (C.C.); and Janssen Research \& Development, San Diego, California (P.W.)
\end{abstract}

Received October 2, 2018; accepted February 4, 2019

\begin{abstract}
In this phase 1 study, the absolute bioavailability and absorption, metabolism, and excretion (AME) of apalutamide, a competitive inhibitor of the androgen receptor, were evaluated in 12 healthy men. Subjects received $240 \mathrm{mg}$ of apalutamide orally plus a 15-minute intravenous infusion of $100 \mu \mathrm{g}$ of apalutamide containing $9.25 \mathrm{kBq}$ ( $250 \mathrm{nCi}$ ) of ${ }^{14} \mathrm{C}$-apalutamide (2 hours postdose) for absolute bioavailability assessment or plus one $400-\mu \mathrm{g}$ capsule containing $37 \mathrm{kBq}$ (1000 nCi) of ${ }^{14} \mathrm{C}$-apalutamide for AME assessment. Content of ${ }^{14} \mathrm{C}$ and metabolite profiling for whole blood, plasma, urine, feces, and expired air samples were analyzed using accelerator mass spectrometry. Apalutamide absolute oral bioavailability was $\approx 100 \%$. After oral
\end{abstract}

administration, apalutamide, its $\mathbf{N}$-desmethyl metabolite (M3), and an inactive carboxylic acid metabolite (M4) accounted for most ${ }^{14} \mathrm{C}$ in plasma $(45 \%, 44 \%$, and $3 \%$, respectively). Apalutamide elimination was slow, with a mean plasma half-life of 151-178 hours. The mean cumulative recovery of total ${ }^{14} \mathrm{C}$ over 70 days postdose was $64.6 \%$ in urine and $24.3 \%$ in feces. The urinary excretion of apalutamide, M3, and M4 was $1.2 \%, 2.7 \%$, and $31.1 \%$ of dose, respectively. Fecal excretion of apalutamide, M3, and M4 was $1.5 \%, 2.0 \%$, and $2.4 \%$ of dose, respectively. Seventeen apalutamide metabolites and six main metabolic clearance pathways were identified. In vitro studies confirmed CYP2C8 and CYP3A4 roles in apalutamide metabolism.

\section{Introduction}

Apalutamide is a potent, specific, orally administered competitive inhibitor of the androgen receptor. Apalutamide has been studied in the treatment of men with nonmetastatic castration-resistant prostate cancer (CRPC) who are at high risk for the development of metastatic disease (Smith et al., 2005; Lin et al., 2017). Its mechanism of action involves the blockade of androgen receptor nuclear translocation, DNA binding to androgen response elements, and transcription of androgen target genes (Clegg et al., 2012). On the basis of the phase 3 SPARTAN trial, which demonstrated that metastasis-free survival was more than 2 years longer with apalutamide $240 \mathrm{mg}$ /day compared with placebo in men with nonmetastatic CRPC (Smith et al., 2018), apalutamide received US Food and Drug Administration approval (ERLEADA, 2018).

In a phase 1 study conducted in men with CRPC, apalutamide was safe and well tolerated across the dose range of 30-480 mg (Rathkopf et al., 2013). The $C_{\max }$ of apalutamide and the area under the plasma concentration-time curve (AUC) increased proportionally after repeated once-daily dosing of 30-480 mg. Apalutamide steady state was achieved after 4 weeks, and the mean accumulation ratio was approximately 5-fold. Apalutamide declined slowly in plasma, and the mean effective

This study was funded by Janssen Research \& Development.

https://doi.org/10.1124/dmd.118.084517.

S This article has supplemental material available at dmd.aspetjournals.org. half-life was about 3-4 days at steady state. An increase in apparent clearance was observed with repeat dosing of apalutamide, likely due to metabolic autoinduction (ERLEADA, 2018). The major active metabolite of apalutamide, $\mathrm{N}$-desmethyl apalutamide (M3), which exhibited one-third the activity of apalutamide in an in vitro transcriptional reporter assay, was detected in plasma as early as 1 hour after ingestion of the first apalutamide dose, with concentrations increasing steadily during the first 24 hours after single-dose administration. M3 plasma levels approached steady state after 6-8 weeks and were comparable to its parent with minimal fluctuation. A minor, inactive carboxylic acid metabolite of apalutamide (M4) was also detected in plasma, with systemic exposure below $10 \%$ of the parent compound apalutamide plus metabolites (M3 and M4) combined at steady state. The recommended phase 2 dose of apalutamide, $240 \mathrm{mg} /$ day, showed robust activity based on durable prostate-specific antigen responses and disease control in a phase 2 study of patients with nonmetastatic CRPC (Smith et al., 2016), findings that have since been confirmed in the phase 3 SPARTAN trial (Smith et al., 2018).

Though previous studies that supported the clinical development of apalutamide have evaluated the efficacy and pharmacodynamic profile of this agent, the absolute bioavailability, absorption, metabolism, and excretion (AME) profile of apalutamide in humans has not been completely characterized. The present study was therefore conducted with the primary objective of determining the absolute bioavailability, and absorption, metabolic pathways, and excretion routes of

ABBREVIATIONS: ABT, aminobenzotriazole; AE, adverse event; AME, absorption, metabolism, and excretion; AMS, accelerator mass

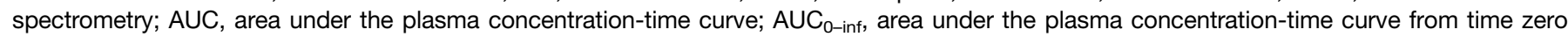
to infinity; $\mathrm{AUC}_{0-\mathrm{t}}$, area under the plasma concentration-time curve from time zero to the last time point with a measurable concentration; BNPP, bis-nitrophenol phosphate; CRPC, castration-resistant prostate cancer; HPLC, high-performance liquid chromatography; LC, liquid chromatography; $\mathrm{M}$, metabolite; $\mathrm{M} 3, \mathrm{~N}$-desmethyl metabolite; M4, carboxylic acid metabolite of apalutamide; MS, mass spectrometry; MS/MS, tandem mass spectrometry; $\mathrm{m} / \mathrm{z}$, mass/charge ratio; NMR, nuclear magnetic resonance; $\mathrm{T}_{\max }$, time to $C_{\max }$. 
apalutamide in healthy men after the administration of a single oral dose of apalutamide. Because of the very low intrinsic clearance of apalutamide, a ${ }^{14} \mathrm{C}$ microtracer approach was used to limit the radioactivity burden to study participants (Lappin, 2016; Guerini et al., 2017; Helmer et al., 2017; Morcos et al., 2017). Based on AME results, in vitro phenotyping of apalutamide was conducted using cryopreserved human hepatocytes to definitively identify the key enzymes involved in its biotransformation.

\section{Materials and Methods}

\section{Clinical AME Study in Healthy Subjects}

Study Design. This was an open-label, phase 1, single-dose, microtracer study to evaluate the AME of ${ }^{14} \mathrm{C}$-labeled apalutamide in healthy men. The study was conducted in two parallel parts, each involving six subjects. Part A was used to determine the absolute oral bioavailability of apalutamide within a single dosing period; part B determined the AME of apalutamide and its metabolites. An independent ethics committee reviewed and approved the study protocol. The study was conducted in accordance with Good Clinical Practice guidelines. All subjects provided their written informed consent.

Study Population. Subjects eligible for inclusion in the study were healthy men $50-80$ years of age with a body mass index of $18.5-30.0 \mathrm{~kg} / \mathrm{m}^{2}$. Subjects with clinically relevant pathology; irregular bowel habit; or hematology, blood, or urine chemistry results outside the normal range were excluded.

Study Medication. In part A, on study day 1, subjects received an oral dose of $240 \mathrm{mg}$ apalutamide in softgel capsules containing a lipid-based solution of apalutamide. This was followed approximately 2 hours postdose by a 15 -minute intravenous infusion of $100 \mu \mathrm{g}$ of apalutamide containing $9.25 \mathrm{kBq}(250 \mathrm{nCi})$ of ${ }^{14} \mathrm{C}$-apalutamide. The position of the ${ }^{14} \mathrm{C}$ label was at the carbonyl group of the diazaspiro octyl ring of apalutamide. The 2-hour dosing interval between oral and intravenous dosing was based on the average time to $C_{\max }\left(\mathrm{T}_{\max }\right)$ and chosen to overcome any time-dependent differences in pharmacokinetics. In part $\mathrm{B}$, on study day 1 , subjects received $240 \mathrm{mg}$ of apalutamide together with one $400-\mu \mathrm{g}$ capsule containing $37 \mathrm{kBq}(1000 \mathrm{nCi})$ of ${ }^{14} \mathrm{C}$-apalutamide in the same lipid vehicle. The maximum allowable radioactivity dose for a microtracer study was chosen. In both parts, the radiation burden to which subjects were exposed was below $0.1 \mathrm{mSv}$ and, as such, no radiation exposure needed to be calculated at the doses of apalutamide used for the study. Oral study medication was administered in the morning after an overnight fast of at least 10 hours, with fasting continuing for 4 hours postdose.

Pharmacokinetic Evaluations. Pharmacokinetic evaluations were undertaken for ${ }^{14} \mathrm{C}$-apalutamide, apalutamide, M3, M4, and total ${ }^{14} \mathrm{C}$; radioactivity and metabolic profiling were conducted at various time points.

In part $\mathrm{A}$, after dosing, the subjects remained in the clinic for 144 hours and returned to the clinic for six ambulatory visits on days 10, 14, 21, 28, 42, and 56 . Blood samples were collected for the determination of plasma pharmacokinetic parameters predose, and $0.5,1,1.5,2,3,4,6,8,10,14,24,48,72,96,120,144$, 216, 312, 480, 648, 984, and 1320 hours postdose. Blood sampling for the assessment of plasma pharmacokinetics of ${ }^{14} \mathrm{C}$-apalutamide were taken predose, at $5,15,20$, and 30 minutes, and at 1, 2, 4, 6, 8, 12, 22, 46, 70, 94, 118, 142, 214, $310,478,646,982$, and 1318 hours postinfusion.

In part B, after dosing, the subjects remained in the clinic for 240 hours and returned to the clinic for six 24-hour clinic periods for the collection of urine, feces, and blood samples on days 14, 21, 28, 42, 56, and 70. Blood samples for total ${ }^{14} \mathrm{C}$ and metabolic profiling in plasma were taken predose, and $0.5,1,1.5,2$, $3,4,6,8,12,24,48,72,120,168,240,336,504,672,1008,1344$, and 1680 hours postdose. Pharmacokinetic assessments and breath tests for total ${ }^{14} \mathrm{C}$ in whole blood and expired air, respectively, were additionally determined from samples taken predose, and 1, 4, 8, 12, 24, 48, 72, 120, and 168 hours postdose. Samples for pharmacokinetic assessments of total ${ }^{14} \mathrm{C}$ and metabolic profiling in urine and feces were taken predose, and at 24-hour intervals on days 1-10, and on days 14-15, 21-22, 28-29, 42-43, 56-57, 70, and 71 (of which day 71 coincided with follow-up).

Sample Preparation for Determination of ${ }^{14} \mathrm{C}$ Content. Whole blood collected at defined intervals was centrifuged to obtain the plasma fraction. Urine and fecal samples were pooled across time intervals. Fecal samples were homogenized, and subsamples of each homogenate were freeze dried. All samples were shipped in dry ice (or, in the case of expired air trapping solution samples, on ice packs) to Xceleron (Germantown, MD) for ${ }^{14} \mathrm{C}$ analysis by accelerator mass spectrometry (AMS). Freeze-dried feces samples were stored at room temperature. Expired air samples were stored at $4^{\circ} \mathrm{C}$, and all other samples were stored at $-80^{\circ} \mathrm{C}$ until required for analysis. Selected samples of plasma, whole blood, and expired air trapping solution underwent liquid scintillation counting to confirm that samples did not require dilution prior to AMS analysis. In the case of urine, for which the relatively high urea content can affect the graphitization process and the ${ }^{14} \mathrm{C} /{ }^{12} \mathrm{C}$ ratio in the graphite produced, samples were first diluted 10-fold with acetonitrile. The carbon content of feces and expired air trapping solution samples was determined using a PerkinElmer (Waltham, MA) 2400 Series 2 C,H,N analyzer; for those samples containing minimal amounts of carbon, sodium benzoate was added as carrier to achieve approximately $2 \mathrm{mg}$ of carbon for graphitization. Plasma, whole blood, urine, expired air trapping solution, and freeze-dried feces samples and controls were placed in glass tubes containing prebaked copper oxide powder and dried under vacuum before analysis.

Metabolite Profiling and Identification in Urine and Feces. Individual samples were pooled across subjects and collection intervals in proportion to the total volume or weight of the excreta collected. Two time-point regions, 0-240 and 240-1680 hours, were profiled; after preparation, samples were fractionated by high-performance liquid chromatography (HPLC), and the resulting fractions were subsequently analyzed by AMS for ${ }^{14} \mathrm{C}$ content at Xceleron. The HPLC column was an XBridge Phenyl 3.5- $\mu \mathrm{m}, 150 \times 3.0 \mathrm{~mm}$ i.d. (Waters, Milford, MA) kept at $30^{\circ} \mathrm{C}$. Mobile phase A consisted of $0.025 \mathrm{M}$ ammonium acetate (adjusted to $\mathrm{pH} 4$ with acetic acid), and mobile phase $\mathrm{B}$ consisted of methanol/acetonitrile 20/80 (v/v). Gradient elution was applied at a mobile phase flow rate of $1 \mathrm{ml} / \mathrm{min}$, and $100-\mu \mathrm{l}$ aliquots of processed urine or feces extract sample were injected. The two time-point regions that had been fractionated by Xceleron for AMS, as well as a selection of HPLC fractions obtained by Xceleron, were also analyzed by ultraperformance liquid chromatography (UPLC)-highresolution mass spectrometry to identify the metabolites in the fractions and to ensure the correct link between identity and radioactive signal as obtained by AMS. The UPLC system consisted of an Acquity Binary Solvent Manager (Waters) and an Acquity injector (Waters); the UPLC column was an XBridge Phenyl $2.5-\mu \mathrm{m}$ column with $150 \times 1.0 \mathrm{~mm}$ i.d. kept at $30^{\circ} \mathrm{C}$ and run using the same mobile phase solvents as for the HPLC method applied by Xceleron. Gradient elution was applied at a mobile phase flow rate of $90 \mu \mathrm{l} / \mathrm{min}$. The mass spectrometer (Synapt G2-S; Waters, Wilmslow, UK) was equipped with a dual electrospray ionization probe and calibrated with a sodium formate solution delivered through the sample spray. The data [obtained by mass spectrometry (MS) and tandem MS (MS/MS)] were acquired in centroid mode with scan times between 0.2 and 0.5 seconds. In MS/MS mode, collision energies of 15, 20, 25, and $35 \mathrm{~V}$ in the transfer cell were used. All data were processed using the Masslynx MS software (Waters).

Sample Preparation for Metabolite Identification in Urine and Feces. Urine was thawed at room temperature and centrifuged. After centrifugation, samples were transferred to HPLC vials and analyzed by LC-MS. Pooled feces homogenates (feces/water ratio, 1:1) were extracted using acetonitrile as extraction solvent. Three milliliters of acetonitrile was added to $1 \mathrm{~g}$ of feces homogenate and vortex mixed. Samples were then centrifuged for 20 minutes at $4^{\circ} \mathrm{C}$ and $2500 \mathrm{rpm}$, whereupon the supernatant was transferred to a new tube. This procedure was repeated twice, so in total $3 \times 3 \mathrm{ml}$ of acetonitrile was used. The combined supernatants were evaporated to dryness under a nitrogen stream at $40^{\circ} \mathrm{C}$ and then stored at $-20^{\circ} \mathrm{C}$ in a freezer until further analysis. The dried extract was reconstituted in $300 \mu \mathrm{l}$ of DMSO, vortex mixed, and sonicated, whereupon $700 \mu 1$ of water was added. Then, the sample was centrifuged, transferred to a liquid chromatography (LC) vial and analyzed by LC-MS. Feces and urine HPLC fractions received from Xceleron were obtained in separate tubes in a 96-well deep-well plate and covered with a self-adhesive seal. The plates were stored frozen until analysis, when the self-adhesive seal was removed and the individual tubes were closed using rubber vial caps. A selection of the tubes was made for subsequent LC-MS analysis, for the purpose of confirming the identity of metabolites in the fractions. The selection of tubes was transferred to a new 96-well plate after thawing and vortex mixing of the tubes, and analyzed by LC-MS.

Targeted Quantification of ${ }^{14} \mathrm{C}$-Apalutamide, Apalutamide, M3, and M4 in Plasma Samples. For targeted quantification of ${ }^{14} \mathrm{C}$-apalutamide, individual plasma samples were fractionated using HPLC after sample preparation. The 
fraction containing ${ }^{14} \mathrm{C}$-apalutamide was collected and subsequently analyzed by AMS for ${ }^{14} \mathrm{C}$ content at Xceleron. For targeted quantification of apalutamide, M3, and M4, individual plasma samples were analyzed after sample preparation using HPLC-MS/MS. The HPLC column was an XBridge C18 3.5- $\mu \mathrm{m}$ column, with $50 \times 2.1 \mathrm{~mm}$ i.d. and kept at $40^{\circ} \mathrm{C}$. Mobile phase A consisted of $0.1 \%$ formic acid, and mobile phase B consisted of acetonitrile. Gradient elution was applied at a mobile phase flow rate of $0.5 \mathrm{ml} / \mathrm{min}$, and $15 \mu \mathrm{l}$ aliquots of processed plasma were injected. The mass spectrometer (API-4000; AB Sciex, Framingham, MA) was equipped with an ionspray source and operated in the negative ion multiple reaction monitoring mode. Mass transitions monitored were $476.0 \rightarrow 419.1$ for apalutamide, $464.0 \rightarrow 323.1$ for M3, and $463.0 \rightarrow 377.2$ for M4. Mass transitions for three internal standards (one for each analyte) were also included.

Sample Preparation for Analysis of Apalutamide, M3, and M4 in Plasma. To each 50- $\mu \mathrm{l}$ plasma sample, $20 \mu \mathrm{l}$ of internal standard mixture and $100 \mu \mathrm{l}$ of $2 \%$ formic acid in water were added and vortex mixed. Next, $750 \mu \mathrm{l}$ of ert-butylmethyl ether was added and vortex mixed for 2 minutes, and subsequently was centrifuged. Next, $250 \mu \mathrm{l}$ of the organic layer was transferred to a new container, and $100 \mu 1$ of $10 \%$ propane diol solution was added then evaporated to dryness at $40^{\circ} \mathrm{C}$ under a nitrogen stream. The residue was redissolved in $250 \mu \mathrm{l}$ of methanol/0.1\% formic acid 30/70 (v/v). A $15-\mu \mathrm{l}$ aliquot was injected and analyzed by LC-MS/MS using an API-4000 Triple Quadrupole Mass Spectrometer (AB Sciex) operated in the multiple reaction monitoring mode, and concentrations of apalutamide, M3, and M4 in the samples were determined.

Safety Evaluations. Safety and tolerability were evaluated based on assessments of adverse events (AEs), vital signs, 12-lead electrocardiogram, clinical laboratory, and physical examination.

Statistical Methods. No prospective calculations of statistical power have been made for this study. The sample size of six subjects in each study part was deemed adequate to provide information regarding safety, tolerability, and pharmacokinetics after single doses of apalutamide. Data were summarized using descriptive statistics for the arithmetic mean, S.D., CV, geometric mean, percentage of $\mathrm{CV}$, and minimum and maximum values.

\section{In Vitro Phenotyping in Human Hepatocytes}

Incubation Procedure for Human Hepatocyte Relay Incubations. Hepatocyte incubations were performed using the relay method described by Di et al. (2012), with minor modifications, as follows. Pooled cryopreserved human hepatocytes from 20 human donors (Celsis IVT, Baltimore, MD) were thawed and subsequently resuspended in Williams' Medium E (Sigma W1878; Millipore Sigma, St. Louis, MO) supplemented with L-glutamine and HEPES. Hepatocytes were added to 24 -well plates at a concentration of $0.5 \times 10^{6}$ cells $/ \mathrm{ml}$ in a final volume of $750 \mu \mathrm{l}$. Next, test compound was spiked at a final concentration of 1 or $5 \mu \mathrm{M}$ in both the presence and absence of diagnostic inhibitors. Substrates and inhibitors were dissolved in methanol (final concentration, $0.1 \%$ ). Plates were covered with Breathe-Easy gas-permeable membranes (MilliporeSigma) and incubated at $37^{\circ} \mathrm{C}$ in a $5 \% \mathrm{CO}_{2}$ incubator, at $75 \%$ relative humidity for 4 hours under continuous agitation on a rotary shaker at $150 \mathrm{rpm}$. At times 0 and 4 hours, $100 \mu \mathrm{l}$ of hepatocyte suspension was removed from the incubation and stored at $-20^{\circ} \mathrm{C}$ for subsequent use in measuring compound concentrations prior to centrifugation. The remainder of the hepatocyte suspension was centrifuged at $2700 \mathrm{~g}$ for 10 minutes at $4^{\circ} \mathrm{C}$, and $550 \mu \mathrm{l}$ of supernatant was transferred to a new 24-well plate and stored at $-20^{\circ} \mathrm{C}$ for subsequent assessment of postcentrifugation compound concentrations and for use in the next relay experiment. For the second relay experiment, supernatant plates were thawed and prewarmed to $37^{\circ} \mathrm{C}$ for 20 minutes. Freshly thawed hepatocytes were added to the samples to give a final cell density of $0.5 \times 10^{6}$ cells $/ \mathrm{ml}$ in a final volume of $750 \mu \mathrm{l}$. Plates were incubated at $37^{\circ} \mathrm{C}$ for 4 hours, then sampled and processed as described for the original incubation.

Four relay experiments were conducted, resulting in a combined total incubation time of 16 hours. Samples from the fourth relay were analyzed, revealing the presence of only M3 and M4 as apalutamide metabolites. Subsequently, the involvement of cytochrome P450 and esterases on the formation of M3 and M4 from apalutamide and on the formation of M4 from M3 was evaluated. Each relay included apalutamide together with the following diagnostic inhibitors of hepatic drug metabolism (Parkinson et al., 2010; Hatfield and Potter, 2011; Sevrioukova and Poulos, 2013; Backman et al., 2016): $1 \mu \mathrm{M}$ itraconazole (CYP3A4); $100 \mu \mathrm{M}$ troleandomycin (CYP3A4); $30 \mu \mathrm{M}$ gemfibrozil plus $10.5 \mu \mathrm{M}$ gemfibrozil glucuronide (CYP2C8); the carboxylesterase inhibitor bis-nitrophenol phosphate (BNPP) at $25 \mu \mathrm{M}$; the pan-CYP inhibitor aminobenzotriazole (ABT) at $1000 \mu \mathrm{M}$; and $25 \mu \mathrm{M}$ BNPP plus $1000 \mu \mathrm{M}$ ABT.

Data Analysis. Data were analyzed in Excel (Microsoft, Redmond, WA) using the method described by Di et al. (2012), with a correction applied for drug lost because of hepatic uptake, nonspecific binding, and dilution according to the following equation:

$$
C_{\text {corrected }}=C_{n, \text { total }} x(\text { recovery })^{n} x\left(\frac{\text { well volume }}{\text { transfer volume }}\right)^{n} x \pi_{i=1}^{n}\left(\frac{C_{i-1, \text { total }}}{C_{i-1, \text { supernatant }}}\right) .
$$

\section{Results}

\section{Clinical AME Study in Healthy Subjects}

Study Participants. Twelve of the 28 men screened for this study were included and received study medication (six subjects in each part of the study). All subjects completed the study and were included in the pharmacokinetic and safety analyses. In part A, the median age was 59 years (age range, 53-64 years), median weight at baseline was $80.5 \mathrm{~kg}$ (range, 71.0-98.2), and median body mass index was $26.3 \mathrm{~kg} / \mathrm{m}^{2}$ (body mass index range, $22.9-29.0 \mathrm{~kg} / \mathrm{m}^{2}$ ). In part $\mathrm{B}$, the median age was 57 years (age range, 52-70 years), median weight at baseline was $81.3 \mathrm{~kg}$ (range, 68.8-89.9 kg), and median body mass index was $23.6 \mathrm{~kg} / \mathrm{m}^{2}$ (body mass index range, $22.5-29.4 \mathrm{~kg} / \mathrm{m}^{2}$ ).

Pharmacokinetic Results in Plasma. In part A, plasma concentrations of apalutamide and ${ }^{14} \mathrm{C}$-apalutamide were detected soon after administration, with $C_{\max }$ reached at approximately 1.0 and 0.25 hours after oral dosing and at the end of intravenous infusion, respectively, followed by a rapid initial decline and a more gradual terminal elimination phase (Fig. 1A). The apalutamide metabolites M3 and M4 were present in quantifiable concentrations from 1 hour after the oral dose, with the concentration of M3 increasing more slowly, reaching $C_{\max }$ at approximately 216 hours postdose compared with 3 hours for M4 (Fig. 1B).

Pharmacokinetic parameters after oral dosing with $240 \mathrm{mg}$ of apalutamide and intravenous dosing with ${ }^{14} \mathrm{C}$-apalutamide from part $\mathrm{A}$ are summarized in Table 1. Exposure as assessed using AUC from time zero to the last time point with a measurable concentration $\left(\mathrm{AUC}_{0-\mathrm{t}}\right)$ was approximately 21 -fold higher for apalutamide ( $185 \mathrm{~h} / \mu \mathrm{g}$ per milliliter) and M3 (183 h/ $\mu \mathrm{g}$ per milliliter) compared with M4 $(8.83 \mathrm{~h} / \mu \mathrm{g}$ per milliliter). The geometric mean AUC from time zero to infinity ( $\mathrm{AUC}_{0 \text {-inf }}$ ) was $200 \mathrm{~h} / \mu \mathrm{g}$ per milliliter after $240 \mathrm{mg}$ oral apalutamide administration and $0.0719 \mathrm{~h} / \mu \mathrm{g}$ per milliliter after intravenous administration of $100 \mu \mathrm{g}$ ${ }^{14} \mathrm{C}$-apalutamide, indicating high absolute bioavailability of apalutamide. The geometric mean $\mathrm{AUC}_{0-\text { inf }}$ was $173 \mathrm{~h} / \mu \mathrm{g}$ per milliliter for the intravenous dose when dose normalized to $240 \mathrm{mg}$.

Pharmacokinetic parameters after a single oral dose of $240 \mathrm{mg}$ of apalutamide administered together with $37 \mathrm{kBq}(1000 \mathrm{nCi})$ ${ }^{14} \mathrm{C}$-apalutamide from part $\mathrm{B}$ are summarized in Table 1 . The plasma concentration-time profiles of apalutamide, M3, and M4 were comparable between parts $\mathrm{A}$ and $\mathrm{B}$. As for part $\mathrm{B}$, exposure to apalutamide and $\mathrm{M} 3$ in terms of $\mathrm{AUC}_{0-\mathrm{t}}$ was at least 15-fold higher than to M4. The systemic exposure for total ${ }^{14} \mathrm{C}$ was the sum of those of apalutamide, M3, and M4. Exposure to apalutamide, M3, and M4 expressed as a ratio relative to total ${ }^{14} \mathrm{C}$ accounted for $42 \%, 41 \%$, and $2.7 \%$ of total ${ }^{14} \mathrm{C}$, respectively, based on $\mathrm{AUC}_{0-\mathrm{t}}$. Arithmetic mean concentration-time profiles for apalutamide in plasma and ${ }^{14} \mathrm{C}$-apalutamide in plasma and whole blood were similar (Fig. 1C). Because most of the ${ }^{14} \mathrm{C}$ signal in plasma could be explained by apalutamide, M3, and M4, no plasma samples were included for AMS/UPLC analysis. For plasma metabolites, targeted quantification of apalutamide, M3, and M4 was considered sufficient. 

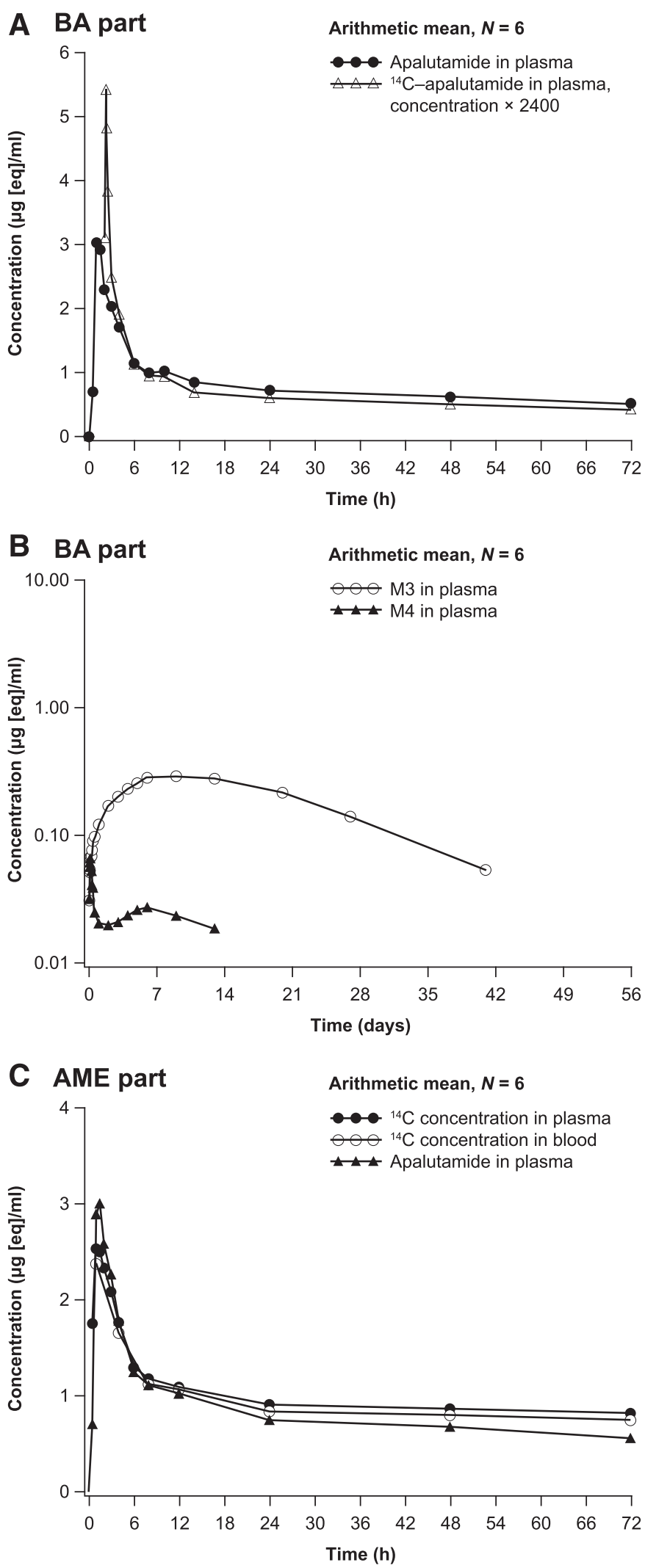

Fig. 1. Arithmetic mean plasma concentration-time profiles: apalutamide and dosenormalized ${ }^{14} \mathrm{C}$-apalutamide in plasma [linear; part A bioavailability (BA) study] (A); apalutamide metabolites M3 and M4 in plasma (logarithmic-linear; BA study) (B); apalutamide in plasma, and ${ }^{14} \mathrm{C}$-apalutamide in plasma and whole blood (linear; part B AME study) (C).
Total ${ }^{14} \mathrm{C}$ in Urine, Feces, and Expired Air. The arithmetic mean cumulative recovery of total ${ }^{14} \mathrm{C}$ in urine, feces, and expired air was $88.9 \%$ (range, 83.8\%-92.7\%) of the administered ${ }^{14} \mathrm{C}$-apalutamide dose by day 71 postdose, from which most of the total ${ }^{14} \mathrm{C}(>80 \%)$ was recovered up to day 42 postdose. Most of the dose was excreted in urine (64.6\%; range, $59.5 \%-71.2 \%$ ) followed by feces (24.3\%; range, $18.1 \%-32.0 \%)$ (Fig. 2). The amount excreted in urine for apalutamide (i.e., unchanged drug), M3, and M4 was $1.20 \%, 2.73 \%$, and $31.12 \%$ of the dose, respectively. Apalutamide was identified as a minor entity, whereas M4 was the major entity excreted in urine. The amounts excreted in feces for apalutamide, M3, and M4 were $1.54 \%, 1.97 \%$, and $2.38 \%$ of the dose, respectively. Apalutamide was identified as a minor entity in feces. Expired air accounted for $<0.06 \%$ of total ${ }^{14} \mathrm{C}$. However, excretion was slow, with just $9.6 \%$ and $0.5 \%$ of the ${ }^{14} \mathrm{C}$ excreted in urine and feces, respectively, within 48 hours of dosing.

Metabolite Identification in Urine and Feces. After a single oral dose of $240 \mathrm{mg}$ of apalutamide, radiochromatograms of urine showed a high concentration of metabolites M4, M19, and M20 throughout the dosing interval (Fig. 3, A and B). In feces, M9, M13, and M14 were the dominant metabolites during the first 240 hours postdose (Fig. 3C), with M13 and M14 also dominant from 240 to 1680 hours postdose (Fig. 3D). The mass balance of unchanged ${ }^{14} \mathrm{C}$-apalutamide and its metabolites in urine and feces after oral administration of $240 \mathrm{mg}$ apalutamide is summarized in Table 2.

Metabolite formation pathways and their retention times are summarized in Table 3. Six main metabolic clearance pathways for apalutamide were identified based on the nature of the observed metabolites. These clearance pathways are described as follows in order of importance (Fig. 4): 1) $\mathrm{N}$ demethylation of the $N$-methylbenzamide moiety to $\mathrm{M} 3$, followed by amide hydrolysis to M4;2) direct amide hydrolysis of the $N$-methylbenzamide moiety to M4 (pathways 1 and 2 taken together are the most important metabolic clearance pathways); 3) ring opening of the diazaspiro octanyl moiety followed by loss of the $N$-(6-cyano-5-(trifluoromethyl) pyridin-3-yl) methanethioamide group (M19), with subsequent $N$-demethylation (M20) and glucuronidation (M21 and M22); 4) nitrile hydrolysis of the cyanopyridinyl moiety (M13), followed by $N$-demethylation (M15) and amide hydrolysis (M18); 5) cysteine condensation with the cyano-pyridinyl moiety (M9) (Berteotti et al., 2014) followed by $N$-demethylation (M14) and amide hydrolysis (M17); and 6) oxidation of the diazaspiro octanyl moiety (M8) followed by $O$-glucuronidation (M11). In contrast, the condensation of cysteine-glycine with the cyano-pyridinyl moiety (M10, M12) and oxidative desulfuration to metabolite (M2) were minor excretion pathways. The cysteinyl glycine adducts M10 and M12 are presumed to be formed via the Pinner reaction (Kalgutkar, 2017). This reaction involves reversible addition of apalutamide to the cysteine of glutathione followed by rearrangement to the more stable cyclic form by enzymatic removal of the glutamyl residue, as described in the literature for odanacatib (Kassahun et al., 2014). The cysteine adducts M9, M14, and M17 can be formed by the reaction of apalutamide to cysteine via the same mechanism or can be formed as downstream metabolites of the cysteine-glycine adducts. Metabolites M9, M10, M12, M14, and M17 are indicative of some intrinsic reactivity of the nitrile function of apalutamide. The reaction scheme for the Pinner reaction is provided in Supplemental Fig. 1. Unchanged drug was mainly excreted in urine, but also was present as a minor entity in feces. M4 was the main metabolite identified in both urine and feces, with M3, M9, M13, M14, M15, M17 (trace), M19, and M20 also common to both routes of elimination.

Safety. The safety profile of apalutamide in parts A and B of this study was similar to that in previous reports, with no serious AEs or discontinuations due to AEs.

\section{In Vitro Phenotyping in Human Hepatocytes}

The turnover of apalutamide in human hepatocytes using an in vitro relay method over 16 hours of incubation was low, with the dynamic range of its intrinsic clearance too low to phenotype the metabolic 
TABLE 1

Summary statistics of pharmacokinetic parameters for apalutamide, $\mathrm{M} 3, \mathrm{M} 4$, and ${ }^{14} \mathrm{C}$-apalutamide in plasma and blood. Geometric mean (\% CV) data are presented, except for $\mathrm{T}_{\max }$ for which median (minimum-maximum) data are presented

\begin{tabular}{|c|c|c|c|c|c|c|c|c|}
\hline Analyte & $C_{\max }$ & $\mathrm{T}_{\max }{ }^{a}$ & $\mathrm{AUC}_{0-\mathrm{t}}$ & $\mathrm{AUC}_{0-\mathrm{inf}}$ & $t_{1 / 2}$ & $\mathrm{~F}$ & $\mathrm{CL}$ & $\mathrm{Vd}$ \\
\hline & $\mu \mathrm{g} / \mathrm{ml}$ & $\mathrm{h}$ & \multicolumn{2}{|c|}{$\mathrm{h} / \mu \mathrm{g}$ per milliliter } & $\mathrm{h}$ & & $1 / h$ & 1 \\
\hline \multicolumn{9}{|c|}{ Part A-bioavailability study, $N=6$} \\
\hline Apalutamide & $3.25(12.2)$ & $1.01(1.00-1.50)$ & $185(16.2)$ & $200(14.5)$ & $175(19.0)$ & $1.11(2.1)$ & N.D. & N.D. \\
\hline M3 & $0.302(20.0)$ & $265.84(144.00-313.70)$ & $183(10.6)$ & $199(12.0)$ & $248(23.7)$ & N.D. & N.D. & N.D. \\
\hline $\mathrm{M} 4^{b}$ & $0.0662(33.2)$ & $3.00(2.00-6.00)$ & $8.83(52.0)$ & N.D. & N.D. & N.D. & N.D. & N.D. \\
\hline${ }^{14} \mathrm{C}$-apalutamide & $0.00235(25.3)$ & $0.23(0.08-0.42)$ & $0.0699(19.7)$ & $0.0719(19.7)$ & $170(23.3)$ & N.D. & $1.33(15.0)$ & $326(17.0)$ \\
\hline DN ${ }^{14} \mathrm{C}$-apalutamide & $5.64(25.3)$ & N.D. & $168(19.7)$ & $173(19.7)$ & N.D. & N.D. & N.D. & N.D. \\
\hline \multicolumn{9}{|c|}{ Part B-AME study, $N=6$} \\
\hline Apalutamide & $3.18(14.5)$ & $1.50(1.00-2.02)$ & $175(23.5)$ & $185(22.1)$ & $148(22.0)$ & N.D. & N.D. & N.D. \\
\hline M3 & $0.322(21.9)$ & $204.00(120.00-240.00)$ & $169(14.0)$ & $186(12.2)$ & $225(23.4)$ & N.D. & N.D. & N.D. \\
\hline $\mathrm{M} 4^{b}$ & $0.0717(19.4)$ & $3.00(2.00-6.00)$ & $11.1(39.0)$ & N.D. & N.D. & N.D. & N.D. & N.D. \\
\hline Total ${ }^{14} \mathrm{C}^{c}$ & $2.82(25.9)$ & $1.25(0.50-2.00)$ & $413(10.2)$ & $418(10.5)$ & 257 (11.6) & N.D. & N.D. & N.D. \\
\hline Total ${ }^{14} \mathrm{C}$ (blood $)^{c, d}$ & $2.34(19.1)$ & $1.00(1.00-1.00)$ & $133(11.1)$ & N.D. & N.D. & N.D. & N.D. & N.D. \\
\hline
\end{tabular}

$\mathrm{CL}$, clearance; DN, dose normalized to $240 \mathrm{mg} ; \mathrm{F}$, absolute bioavailability; $N$, number of subjects; N.D., not determined; $t_{1 / 2}$, terminal half-life; Vd, volume of distribution.

${ }^{a}$ For ${ }^{14} \mathrm{C}$-apalutamide, $\mathrm{T}_{\max }$ was measured relative to the start of the intravenous infusion at $2 \mathrm{~h}$ after oral dosing.

${ }^{b}$ Geometric mean $\mathrm{AUC}_{0-\text { inf }}$ and $t_{1 / 2}$ could not be reliably determined.

${ }^{c}$ For total ${ }^{14} \mathrm{C}$ in plasma and blood, the units for $C_{\max }(\mu \mathrm{g} \mathrm{Eq} / \mathrm{ml}), \mathrm{AUC}_{0-\mathrm{t}}\left(\mathrm{h} / \mu \mathrm{g}\right.$ Eq per milliliter), and $\mathrm{AUC} \mathrm{C}_{0 \text {-inf }}(\mathrm{h} / \mu \mathrm{g}$ Eq per milliliter) differed from the units shown above.

${ }^{d}$ For total ${ }^{14} \mathrm{C}$ in blood, $\mathrm{AUC}_{0-\mathrm{t}}$ was calculated up to time $t=168 \mathrm{~h}$.

enzymes involved. Therefore, the involvement of cytochrome P450 and esterases in the metabolism of apalutamide was determined based on the formation of M3 and M4 in the presence of inhibitors. After four relays, gemfibrozil, itraconazole, and troleandomycin inhibited the formation of M3 by $55 \%, 17 \%$, and $23 \%$, respectively (Fig. 5A). BNPP resulted in a $42 \%$ reduction in $\mathrm{M} 3$, whereas BNPP plus ABT completely inhibited the metabolism of apalutamide to M3 (Fig. 5A). Gemfibrozil, itraconazole, and troleandomycin had no significant inhibition effects on the formation of M4 (Fig. 5B). No metabolite formation was observed for BNPP or BNPP plus ABT (Fig. 5B). The conversion of M3 to M4 was mediated almost exclusively by esterases, since the formation of M4, after four relays involving the incubation of M3, was completely inhibited by BNPP alone, and only inhibited to a minor extent by ABT.

\section{Metabolite Structure Elucidation by LC-MS/MS}

The identified metabolites, diagnostic product ions, and theoretical exact $\mathrm{m} / \mathrm{z}$ are presented in Table 3. The identity of metabolites M3, M2, and M4 were additionally confirmed by chromatography with a reference standard. The identity of M9 was confirmed by two-dimensional nuclear magnetic resonance (NMR) spectroscopy analysis. Details of the structural elucidation of apalutamide and its metabolites by multiplestage MS analyses are described in the following sections; the proposed structures of detected metabolites were used to postulate the in vivo metabolic pathways for apalutamide in humans (Fig. 4). Mass spectra of unchanged drug and major human plasma metabolites are shown in Figs. 6 and 7. Further supporting information for the proposed structures and diagnostic product ions of M19 (Supplemental Fig. 2) and M10 (Supplemental Fig. 3) are included in the Supplemental Material.

\section{Apalutamide}

The accurate full scan and product ion mass spectra of apalutamide are shown in Fig. 6. Full-scan mass analysis gave an $[\mathrm{M}+\mathrm{H}]^{+}$at $\mathrm{m} / \mathrm{z}$ $478.0946\left(\mathrm{C}_{21} \mathrm{H}_{16} \mathrm{~N}_{5} \mathrm{O}_{2} \mathrm{~F}_{4} \mathrm{~S} ; 14.5\right.$ rings plus double bonds; $\left.-3.1 \mathrm{ppm}\right)$. A proposed structure of the main apalutamide product ions is shown in Supplemental Fig. 4.

\section{M2}

The $[\mathrm{M}+\mathrm{H}]^{+}$of $\mathrm{M} 2$ at $\mathrm{m} / z, 462$ was 16 Da lower than that of apalutamide. The calculated elemental composition obtained by exact mass measurement corresponds to oxidative desulfuration of the parent drug. The shifted accurate mass product ions at $m / z 434(450-16)$, 403 (419-16), and $214(230-16)$, and unchanged ions at $m / z, 193$ and 162 were consistent with this structure assignment.

\section{M3}

The $[\mathrm{M}+\mathrm{H}]^{+}$of $\mathrm{M} 3$ at $m / z 464$ was 14 Da lower than that of apalutamide. The calculated elemental composition obtained by exact mass measurement corresponds to the loss of $1 \mathrm{C}$ and $2 \mathrm{H}$ from the parent drug, pointing to demethylation of the $\mathrm{N}$-methylbenzamide moiety. Diagnostic accurate mass product ions at $m / z 436(450-14), 207(221-14)$, and $179(193-14)$ as well as unchanged ions at $\mathrm{m} / \mathrm{z} 447,419,190$, and 162 were consistent with this structure.

\section{M4}

The $[\mathrm{M}+\mathrm{H}]^{+}$of M4 at $\mathrm{m} / \mathrm{z} 465$ was $13 \mathrm{Da}$ lower than that of apalutamide. The calculated elemental composition obtained by exact mass measurement corresponds to the loss of $1 \mathrm{C}, 3 \mathrm{H}$, and $1 \mathrm{~N}$, and the gain of $1 \mathrm{O}$ relative to the parent drug. The unchanged ions at $\mathrm{m} / \mathrm{z}, 230$ and

\section{${ }^{14} \mathrm{C}$ Recovery}

Arithmetic mean, $N=6$

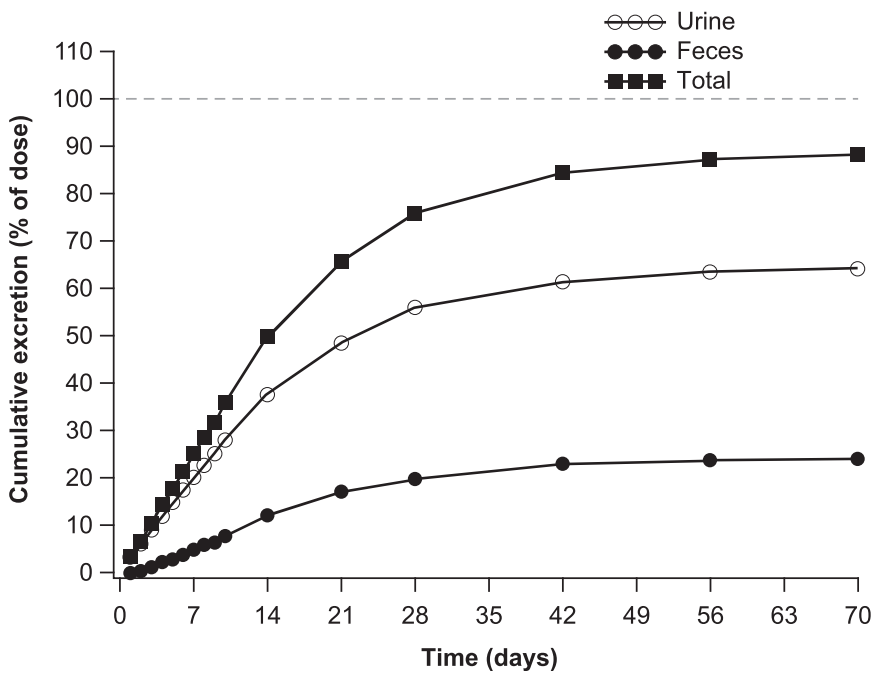

Fig. 2. Cumulative recovery of ${ }^{14} \mathrm{C}$ in urine, feces, and total excretion. 
A

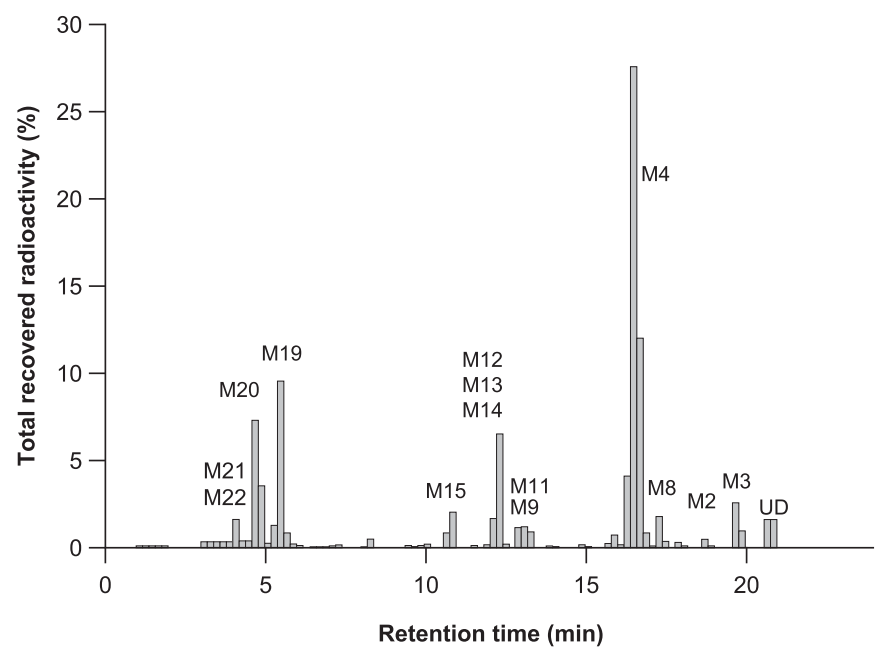

C

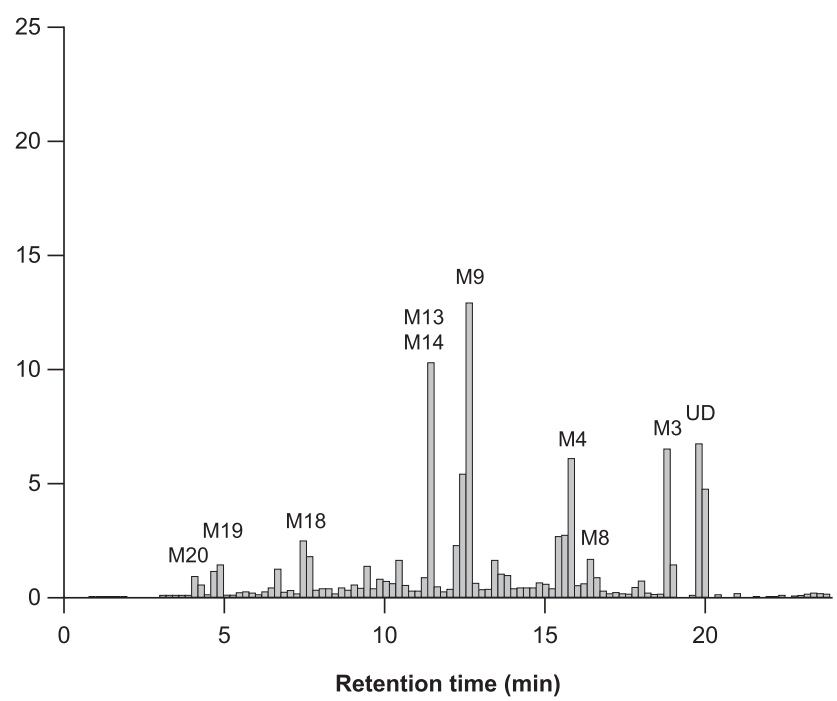

B

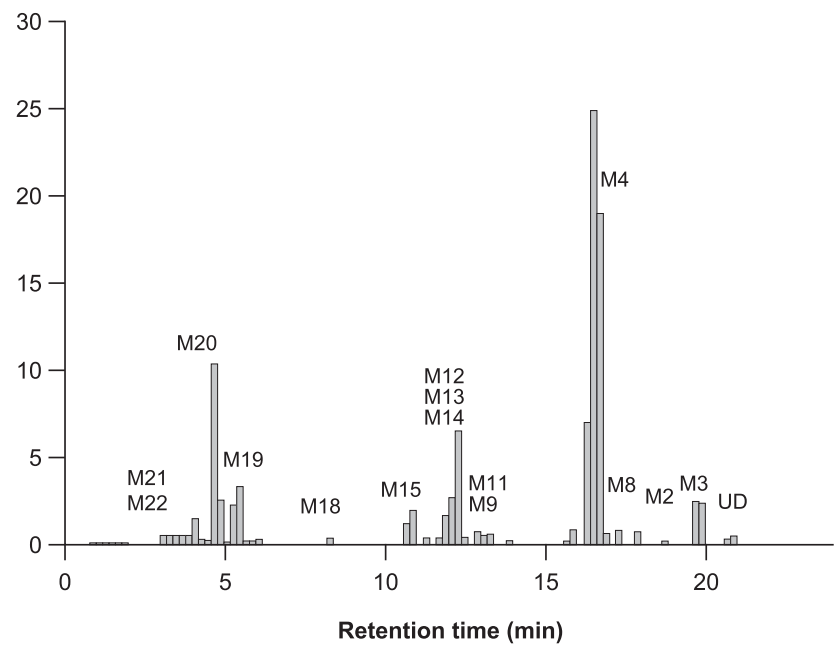

D

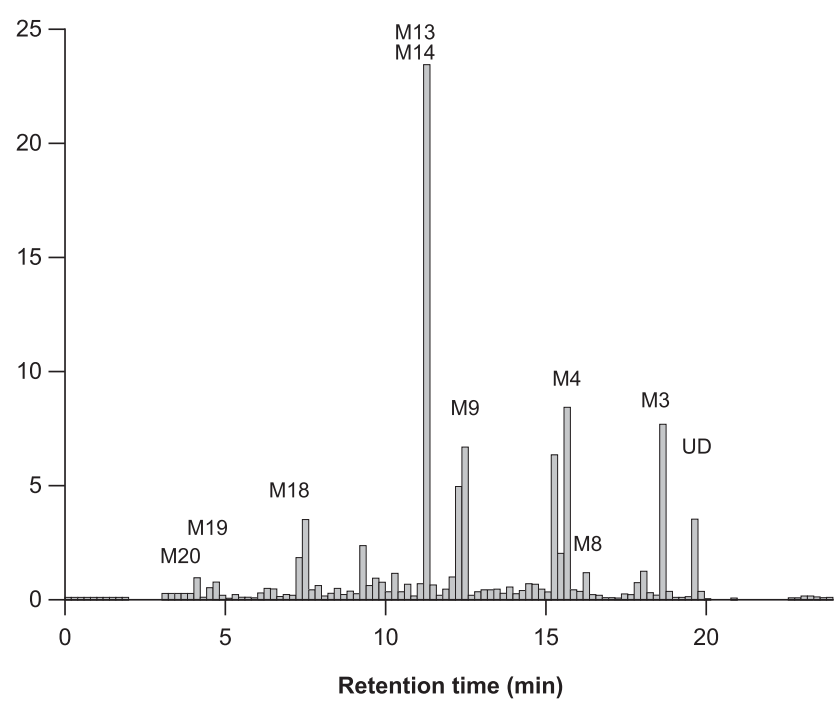

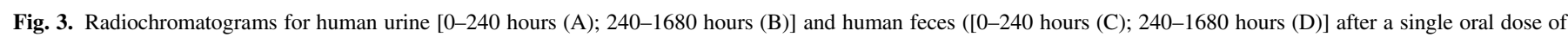
$240 \mathrm{mg}$ of apalutamide.

162 indicate that the site of metabolism is at the $N$-methylbenzamide part of the molecule. Based on the elemental composition change and the site of metabolism, the $N$-methylbenzamide moiety is metabolized to a carboxylic acid. The accurate mass product ions at $m / z 437$ (450 - 13), $208(221-13)$, and $180(193-13)$ are consistent with this structure assignment.

\section{M8}

The $[\mathrm{M}+\mathrm{H}]^{+}$of M8 at $\mathrm{m} / z, 494$ was 16 Da higher than that of apalutamide. The calculated elemental composition obtained by exact mass measurement corresponds to oxidation $(+1 \mathrm{O})$ of the parent drug. The shifted accurate mass product ions at $m / z, 219\left(221+16 \mathrm{H}_{2} \mathrm{O}\right)$ and $206(190+16)$ and the unchanged ions at $\mathrm{m} / \mathrm{z}, 450,419,381,230,211$, 193, and 162 indicate that the oxidation should be located at the diazaspirooctanyl moiety.

\section{M9}

The $[\mathrm{M}+\mathrm{H}]^{+}$of M9 at $\mathrm{m} / z 582$ was $104 \mathrm{Da}$ higher than that of apalutamide. The calculated elemental composition obtained by exact mass measurement corresponds to the addition of $\mathrm{C}_{3} \mathrm{H}_{4} \mathrm{O}_{2} \mathrm{~S}$ to the parent drug. The unchanged ion at $m / z, 249$ and the shifted ion at $m / z, 334(230+$ 104) proves that metabolism took place at the $\mathrm{N}$-(6-cyano-5(trifluoromethyl)pyridin-3-yl)) part of the molecule. This, in combination with the elemental composition change and the presence of a cysteinyl-glycine condensation product (metabolite M10; +57 U [glycine] relative to M9) in the same sample point to a condensation of cysteine with the cyano-pyridinyl moiety of the parent drug (Maltsev et al., 2013; Berteotti et al., 2014). The complementary ions at $\mathrm{m} / \mathrm{z}$. 478 and 105.0007 [mercaptopropanoic acid $\left(\mathrm{C}_{3} \mathrm{H}_{5} \mathrm{O}_{2} \mathrm{~S}\right) ; 0.2 \mathrm{mDa}$ error] are in agreement with this structure assignment. The structure as shown in Fig. 4 was assigned. M9 was synthesized following Maltsev et al. (2013), by reacting $1 \mathrm{Eq}$ of apalutamide with $1.5 \mathrm{Eq}$ cysteine, $1.5 \mathrm{Eq}$ $\mathrm{NaHCO}_{3}$, and a catalytic amount of $1 \mathrm{M} \mathrm{NaOH}$ in methanol/water for 24 hours at room temperature under continuous stirring. The sample was neutralized with $1 \mathrm{M} \mathrm{HCl}$ prior to isolation using preparative HPLC. The structure was confirmed by one-dimensional ${ }^{1} \mathrm{H}$ and ${ }^{13} \mathrm{C}$ NMR analysis as well as two-dimensional correlation spectroscopy, heteronuclear single-quantum coherence spectroscopy, and heteronuclear multiple-bond connectivity NMR analysis of the synthesized M9 (data on file). 
TABLE 2

Mass balance of unchanged ${ }^{14} \mathrm{C}$-apalutamide and its metabolites expressed as a percentage of the administered dose and found in urine and feces after oral administration of $240 \mathrm{mg}$ of apalutamide

\begin{tabular}{|c|c|c|c|c|c|c|c|c|c|c|}
\hline \multirow{3}{*}{ Component } & \multicolumn{5}{|c|}{ Urine } & \multicolumn{5}{|c|}{ Feces } \\
\hline & \multicolumn{2}{|c|}{$0-240 \mathrm{~h}$} & \multicolumn{2}{|c|}{$240-1680 \mathrm{~h}$} & \multirow{2}{*}{$\frac{0-1680 \mathrm{~h}}{\% \text { Dose }}$} & \multicolumn{2}{|c|}{$0-240 \mathrm{~h}$} & \multicolumn{2}{|c|}{$240-1680 \mathrm{~h}$} & \multirow{2}{*}{$\frac{0-1680 \mathrm{~h}}{\% \text { Dose }}$} \\
\hline & $\%$ Inj & $\%$ Dose & $\%$ Inj & $\%$ Dose & & $\%$ Inj & $\%$ Dose & $\%$ Inj & $\%$ Dose & \\
\hline M2 & 0.6 & 0.17 & 0.2 & 0.07 & 0.23 & & & & & \\
\hline M3 & 3.5 & 0.98 & 4.8 & 1.75 & 2.73 & 7.9 & 0.62 & 8.3 & 1.35 & 1.97 \\
\hline M4 & 44.6 & 12.53 & 51.5 & 18.59 & 31.12 & 8.8 & 0.69 & 10.4 & 1.69 & 2.38 \\
\hline M8 & 2.2 & 0.61 & 0.8 & 0.29 & 0.90 & 2.5 & 0.20 & 1.2 & 0.19 & 0.39 \\
\hline M9 & 1.0 & 0.29 & 0.6 & 0.21 & 0.50 & 20.5 & 1.61 & 11.6 & 1.88 & 3.49 \\
\hline M10 & Trace & Trace & Trace & Trace & Trace & & & & & \\
\hline M11 & 2.4 & 0.66 & 1.2 & 0.45 & 1.11 & & & & & \\
\hline $\mathrm{M} 12+\mathrm{M} 13+\mathrm{M} 14$ & 8.4 & 2.35 & 6.6 & 2.38 & 4.73 & $11.1^{a}$ & $0.87^{a}$ & $23.4^{a}$ & $3.79^{a}$ & $4.67^{a}$ \\
\hline M15 & 3.0 & 0.84 & 2.0 & 0.71 & 1.54 & Trace & Trace & Trace & Trace & Trace \\
\hline M17 & Trace & Trace & Trace & Trace & Trace & Trace & Trace & Trace & Trace & Trace \\
\hline M18 & 0.5 & 0.14 & 0.4 & 0.13 & 0.27 & 4.2 & 0.33 & 3.5 & 0.57 & 0.90 \\
\hline M19 & 11.7 & 3.29 & 5.6 & 2.02 & 5.31 & 2.5 & 0.20 & 1.3 & 0.21 & 0.41 \\
\hline M20 & 7.7 & 2.17 & 13.0 & 4.68 & 6.85 & 1.4 & 0.11 & 1.1 & 0.17 & 0.29 \\
\hline $\mathrm{M} 21+\mathrm{M} 22$ & 3.2 & 0.91 & 4.2 & 1.53 & 2.44 & & & & & \\
\hline Unchanged drug & 3.3 & 0.92 & 0.8 & 0.28 & 1.20 & 11.5 & 0.91 & 3.9 & 0.64 & 1.54 \\
\hline Sum of reported entities & 92.0 & 25.9 & 91.2 & 33.0 & 58.8 & 70.5 & 5.5 & 64.8 & 10.5 & 16.0 \\
\hline Sum of observed metabolites (dose $\%$ ) & & 28.1 & & 36.1 & 64.2 & & 7.9 & & 16.2 & 24.1 \\
\hline
\end{tabular}

$\%$ Dose, percentage of oral dose; \% Inj, percentage of ${ }^{14} \mathrm{C}$ injected onto the HPLC column.

${ }^{a}$ Includes M13 + M14 only.

\section{M10}

The $[\mathrm{M}+\mathrm{H}]^{+}$of M10 at $\mathrm{m} / z 639$ was $161 \mathrm{Da}$ higher than that of apalutamide. The calculated elemental composition obtained by exact mass measurement corresponds to the addition of $\mathrm{C}_{5} \mathrm{H}_{7} \mathrm{NO}_{3} \mathrm{~S}$ to the parent drug. The unchanged ion at $\mathrm{m} / \mathrm{z}, 249$ and the shifted ion at $\mathrm{m} / \mathrm{z}$ $391(230+161)$ proves that metabolism took place at the $N$ - $(6$-cyano-5(trifluoromethyl)pyridin-3-yl)) part of the molecule. This, in combination with the elemental composition change and the complementary ions at $\mathrm{m} / \mathrm{z} 478$ and 162 (162.022, not to be confused with 162.035 from apalutamide fragmentation; see Supplemental Fig. 3) point to a condensation of cysteine-glycine with the cyano-pyridinyl moiety of the parent drug (Maltsev et al., 2013). Also, the fragment ions at $\mathrm{m} / \mathrm{z} 536$, 508, and 116 (see Supplemental Fig. 3) are in agreement with this structure.

\section{M11}

The $[\mathrm{M}+\mathrm{H}]^{+}$of M11 at $\mathrm{m} / \mathrm{z} 670$ was $176 \mathrm{Da}$ higher than that of M8. The calculated elemental composition points to glucuronidation of M8. A fragment at $m / z, 494$ is formed via in-source fragmentation. When $\mathrm{MS}^{2}$ is performed on either $\mathrm{m} / \mathrm{z}, 670$ or on the in-source fragmentation product

TABLE 3

Identification of in vivo metabolites of apalutamide after a single oral dose of $240 \mathrm{mg}$ in healthy men

\begin{tabular}{|c|c|c|c|c|c|c|c|}
\hline Component & Identity & $\begin{array}{l}\text { Retention Time } \\
\text { LC-AMS } \\
\text { (min) Urine }\end{array}$ & $\begin{array}{l}\text { Retention Time } \\
\text { LC-AMS } \\
\text { (min) Feces }\end{array}$ & $\begin{array}{l}\text { Retention Time } \\
\text { LC-MS (min) }\end{array}$ & $\mathrm{m} / \mathrm{z}$ & Typical MS Fragments & Matrix \\
\hline
\end{tabular}

$\begin{array}{ll}\text { M2 } & -16(-\mathrm{S}+\mathrm{O}) \\ \text { M3 } & -14(\text {-demethylation) } \\ \text { M4 } & -13 \text { (oxidative deamination) } \\ \text { M8 } & +16(+\mathrm{O}) \\ \text { M9 } & +104 \text { (cysteine condensation) } \\ \text { M10 } & +161 \text { (cysteine-glycine condensation) } \\ \text { M11 } & +192 \text { (+O+gluc) } \\ \text { M12 } & +147 \text { (cysteine-glycine addition }+N- \\ & \text { demethylation) } \\ \text { M13 } & +18 \text { (water addition) } \\ \text { M14 } & +90 \text { (cysteine addition+N-demethylation) } \\ \text { M15 } & +4 \text { (water addition+ } N \text {-demethylation) } \\ \text { M17 } & +91 \text { (cysteine addition+oxidative } \\ & \text { deamination) } \\ \text { M18 } & +5 \text { (water addition+oxidative } \\ & \text { deamination) } \\ \text { M19 } & -211 \text { (ring opening+hydrolysis) } \\ \text { M20 } & -225 \text { (ring opening+hydrolysis+ } \\ & \text { demethylation) } \\ \text { M21 } & -35 \text { (ring opening+hydrolysis+gluc) } \\ \text { M22 } & -49 \text { (ring opening+hydrolysis+ } \\ & \text { demethylation+gluc) } \\ \text { UD } & \text { Parent }\end{array}$

\begin{tabular}{|c|c|c|c|c|c|}
\hline $18.7-18.9$ & & 23.0 & 462.1189 & $162,193,214,403,434$ & $\mathrm{U}$ \\
\hline $19.7-19.9$ & $18.7-18.9$ & 24.1 & 464.0804 & $162,179,190,207,419,436,447$ & $\mathrm{~F}, \mathrm{U}, \mathrm{P}$ \\
\hline $16.3-16.9$ & 15.7 & 21.1 & 465.0644 & $162,180,208,230,437$ & $\mathrm{~F}, \mathrm{U}, \mathrm{P}$ \\
\hline $17.3-17.5$ & 16.3 & 21.7 & 494.091 & $162,193,206,211,219,230,381,419,450$ & $\mathrm{~F}, \mathrm{U}$ \\
\hline \multirow[t]{2}{*}{$13.3-13.5$} & 12.5 & 17.7 & 582.0893 & $105,249,334,478,508$ & $\mathrm{~F}, \mathrm{U}$ \\
\hline & & 17.9 & 639.1107 & $116,162,249,391,478,508,536$ & $\mathrm{U}$ \\
\hline $12.9-13.1$ & & 17.4 & 670.1231 & $162,193,219,381,419,450,494$ & $\mathrm{U}$ \\
\hline 12.3 & & 16.8 & 625.0951 & $116,162,235$ & $\mathrm{U}$ \\
\hline 12.3 & 11.3 & 16.5 & 496.1066 & $231,248,249,479$ & $\mathrm{~F}, \mathrm{U}$ \\
\hline 12.1 & 11.3 & 16.5 & 568.0736 & $105,235,334$ & $\mathrm{~F}, \mathrm{U}$ \\
\hline \multirow[t]{2}{*}{10.9} & & 15.1 & 482.091 & $218,235,248,249,409,454,465$ & $\mathrm{~F}, \mathrm{U}$ \\
\hline & & 14.6 & 569.0576 & $105,208,236,334$ & $\mathrm{~F}, \mathrm{U}$ \\
\hline 8.3 & 7.5 & 12.7 & 483.075 & 236,466 & $\mathrm{~F}, \mathrm{U}$ \\
\hline 5.5 & 4.7 & 9.7 & 267.1145 & $58,99,138,162,190,193,208,236,239$ & $\mathrm{~F}, \mathrm{U}$ \\
\hline 4.7 & $4.1-4.3$ & 8.9 & 253.0988 & $138,162,179,190,208,225$ & $\mathrm{~F}, \mathrm{U}$ \\
\hline $3.1-4.1$ & & 8.4 & 443.1466 & $162,208,221,236,239,267$ & $\mathrm{U}$ \\
\hline $3.1-4.1$ & & 8.1 & 429.1309 & $162,208,225,236,253$ & $\mathrm{U}$ \\
\hline $20.7-20.9$ & $19.7-19.9$ & 25.1 & 478.0961 & $162,164,190,193,221,230,419,447,450$ & $\mathrm{~F}, \mathrm{U}, \mathrm{P}$ \\
\hline
\end{tabular}

UD, unchanged drug. 


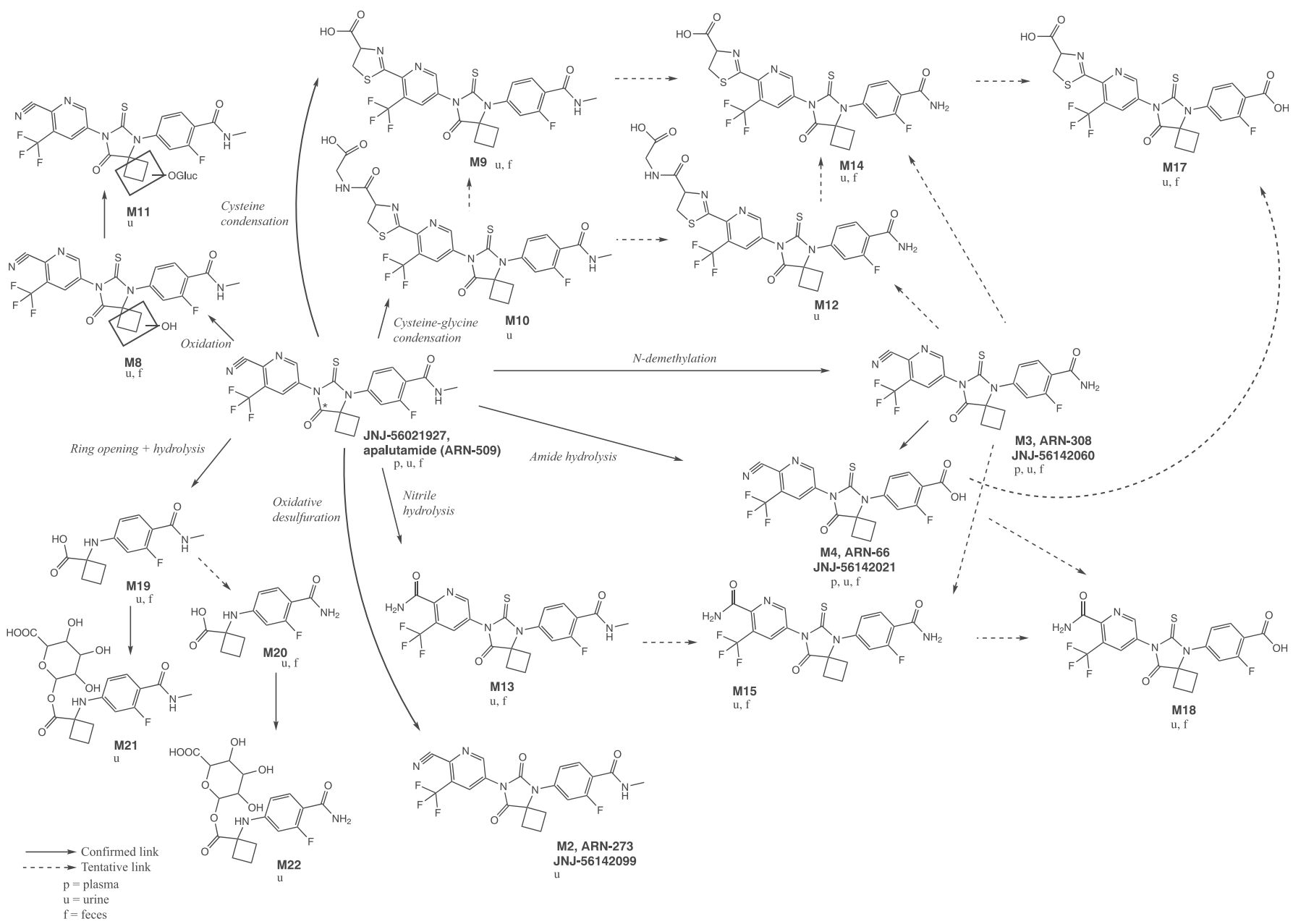

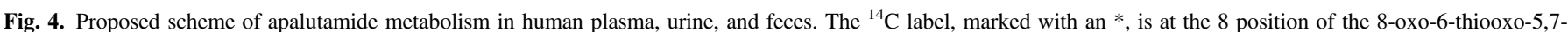
diazaspiro[3,4]octan-5-yl ring.

at $m / z$ 494, then the resulting $\mathrm{MS}^{2}$ spectrum shows high similarity with the $\mathrm{MS}^{2}$ spectrum of $\mathrm{M} 8$, with diagnostic accurate mass product ions at $m / z, 450,419,381,219\left(221+16-\mathrm{H}_{2} \mathrm{O}\right), 193$, and 162.

\section{M12}

The $[\mathrm{M}+\mathrm{H}]^{+}$of M12 at $\mathrm{m} / z, 625$ is 14 Da lower than that of M10. The calculated elemental composition as well as the $-14-\mathrm{U}$ shift of the ion at $\mathrm{m} / z, 235(249-14)$ point to the demethylation at the $N$-methylbenzamide moiety. The ions at $\mathrm{m} / \mathrm{z}, 162$ and 116 are unchanged compared with the M10 fragmentation and confirm the cysteinyl-glycine condensation. The structure as shown in Fig. 4 was assigned.

\section{M13}

The $[\mathrm{M}+\mathrm{H}]^{+}$of M13 at $\mathrm{m} / \mathrm{z} 496$ was $18 \mathrm{Da}$ higher than that of apalutamide. The calculated elemental composition obtained by exact mass measurement corresponds to the addition of $\mathrm{H}_{2} \mathrm{O}$ relative to the parent drug. The unchanged ion at $\mathrm{m} / \mathrm{z}, 249$ and the shifted ion at $\mathrm{m} / \mathrm{z} 248(230+18)$ demonstrate that the addition of $\mathrm{H}_{2} \mathrm{O}$ took place at the $N$-(6-cyano-5-(trifluoromethyl)pyridin-3-yl)) part of the molecule. The neutral loss of ammonia from the parent ion and from the ion at $\mathrm{m} / \mathrm{z} 248$, resulting in the base peak at $\mathrm{m} / \mathrm{z}$, 479 and the fragment at $\mathrm{m} / \mathrm{z}, 231$, respectively, is characteristic for the presence of a primary amine or amide in the structure. Therefore, M13 should be the carboxamide metabolite formed by hydrolysis of the nitrile function.

\section{M14}

The $[\mathrm{M}+\mathrm{H}]^{+}$of M14 at $\mathrm{m} / z, 568$ was $14 \mathrm{Da}$ lower than that of M9, pointing to the demethylation of M9. The calculated elemental composition and the $-14-\mathrm{U}$ shift of the ion at $\mathrm{m} / \mathrm{z} 235(249-14)$ confirm the demethylation at the $N$-methylbenzamide moiety. The ions at $m / z 334$ and 105 are unchanged compared with the M9 fragmentation and confirm the cysteine addition.

\section{M15}

The $[\mathrm{M}+\mathrm{H}]^{+}$of M15 at $\mathrm{m} / z, 482$ was 14 Da lower than that of M13. The calculated elemental composition and the $-14-\mathrm{U}$ shift of the ion at $m / z 235(249-14)$ point to an additional demethylation of the $N$-methylbenzamide moiety. M15 is likely to be a downstream demethylated metabolite from M13, as was also confirmed by the same ions at $m / z, 248(230+18)$ and a neutral loss of ammonia from the latter giving rise to the fragment at $m / z 231$.

\section{M17}

The $[\mathrm{M}+\mathrm{H}]^{+}$of M17 at $\mathrm{m} / z 569$ was 13 Da lower than that of M9. The calculated elemental composition obtained by exact mass measurement corresponds to the loss of $1 \mathrm{C}, 3 \mathrm{H}$, and $1 \mathrm{~N}$ and addition of $1 \mathrm{O}$ relative to M9. The mercaptopropanoic acid ion at $\mathrm{m} / \mathrm{z}, 105$ and the shift of the ion at $m / z, 334(230+104)$ are consistent with the cysteine condensation reaction at the $\mathrm{N}$-(6-cyano-5-(trifluoromethyl)pyridin-3-yl)) part of the 

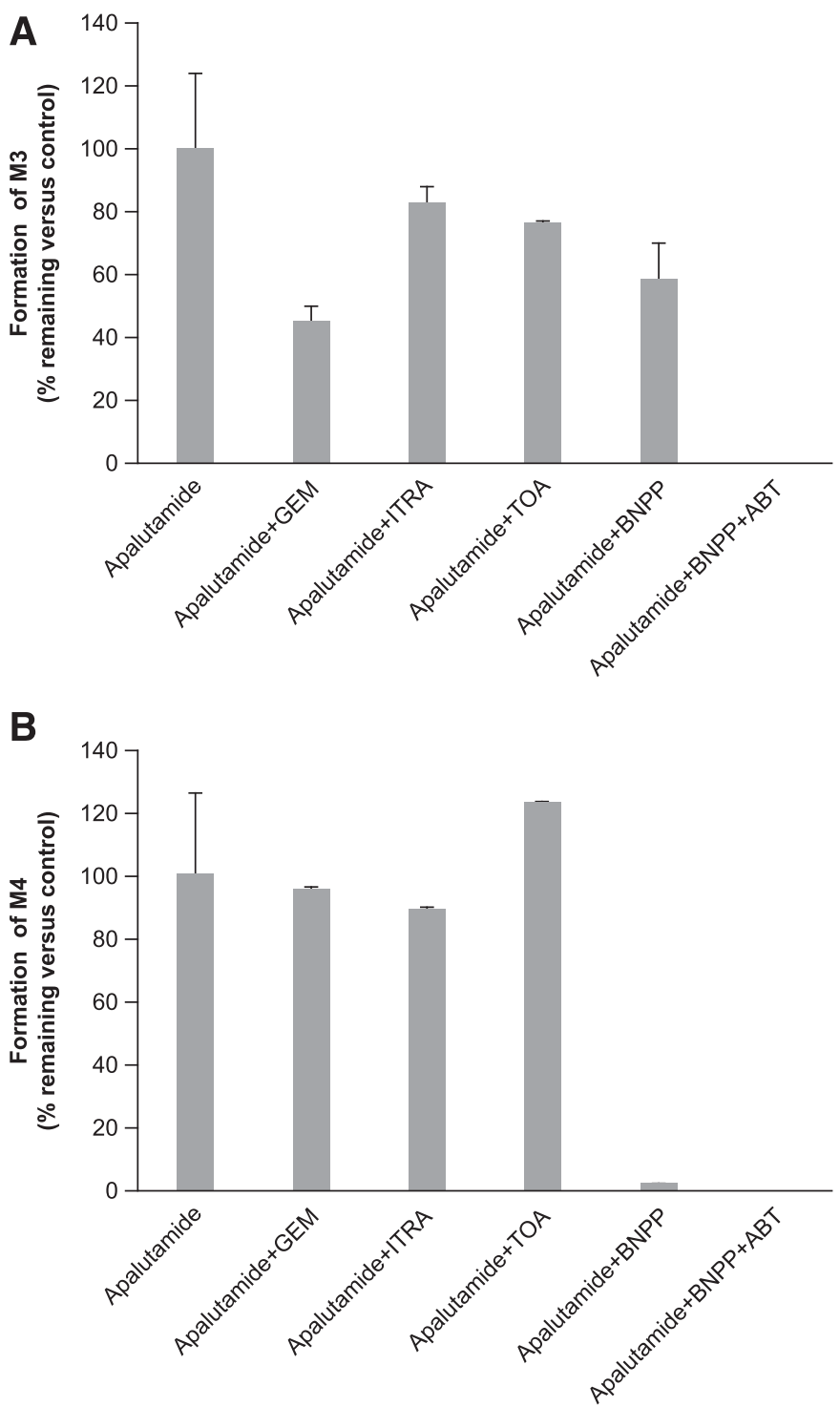

Fig. 5. Effect of specific esterase and cytochrome $\mathrm{P} 450$ inhibitors on the formation of M3 (A) or M4 (B) from apalutamide in human hepatocytes. GEM, gemfibrozil + gemfibrozil glucuronide; ITRA, itraconazole; TOA, troleandomycin.

molecule. The $-13-\mathrm{U}$ shifts of the ions at $\mathrm{m} / \mathrm{z} 208(221-13)$ and $236(249-13)$ are consistent with oxidative deamidation at the $N$-methylbenzamide.

\section{M18}

The $[\mathrm{M}+\mathrm{H}]^{+}$of M18 at $\mathrm{m} / \mathrm{z} 483$ was 13 Da lower than that of M13. The calculated elemental composition obtained by exact mass measurement corresponds to the addition of $2 \mathrm{O}$ and the loss of $1 \mathrm{C}, 1 \mathrm{H}$, and $1 \mathrm{~N}$ relative to the parent drug. The $-13-\mathrm{U}$ shift of the ion at $\mathrm{m} / \mathrm{z}, 236(249-13)$ points to oxidative deamidation of the $\mathrm{N}$-methylbenzamide to a carboxylic acid $\left(-\mathrm{CH}_{3} \mathrm{~N}+\mathrm{O}\right)$. The remaining elemental composition change indicates an $\mathrm{H}_{2} \mathrm{O}$ addition to the other side of the molecule. The neutral loss of ammonia from the parent ion, giving rise to the base peak ion at $m / z 466$, points to the presence of a primary amine or amide. The neutral loss of $\mathrm{CO}_{2}$ in the negative ion mode $\mathrm{MS}^{2}$ spectrum, giving rise to the ion at $\mathrm{m} / \mathrm{z} 437$, is characteristic for an aromatic carboxylic acid. Therefore, a metabolite structure resulting from nitrile hydrolysis to a carboxamide and deamidation of the $\mathrm{N}$-methylbenzamide to a carboxylic acid was assigned.

\section{M19}

The $[\mathrm{M}+\mathrm{H}]^{+}$of M19 at $\mathrm{m} / z, 267$ was $211 \mathrm{Da}$ lower than that of apalutamide. The calculated elemental composition obtained by exact mass measurement corresponds to the addition of $1 \mathrm{O}$ and loss of $\mathrm{C}_{8} \mathrm{~F}_{3} \mathrm{~N}_{3} \mathrm{~S}$, which can metabolically be explained by ring opening of the diazaspirooctanyl moiety followed by loss of the $\mathrm{N}$-(6-cyano-5(trifluoromethyl)pyridin-3-yl)) methanethioamide group. A proposed structure of the main M19 product ions is shown in Supplemental Fig. 2.

\section{M20}

The $[\mathrm{M}+\mathrm{H}]^{+}$of M20 at $m / z, 253$ was 14 Da lower than that of M19. The calculated elemental composition points to a demethylation of metabolite M19. The $\mathrm{MS}^{2}$ spectrum of M20 shows high similarity with that of M19 with accurate mass product ions at $m / z 225(239-14)$ and $179(193-14)$ that have shifted because of the demethylation, whereas the ions at $\mathrm{m} / \mathrm{z}$ 236, 208, 190, 162, and 138 are unchanged relative to M19.

\section{M21}

The $[\mathrm{M}+\mathrm{H}]^{+}$of M21 at $\mathrm{m} / \mathrm{z}, 443$ was 176 Da higher than that of M19. The calculated elemental composition points to glucuronidation of M19. Diagnostic accurate mass product ions at $\mathrm{m} / \mathrm{z}, 267,239,236,208$, and 162 (Supplemental Fig. 4) and at $m / z 221$ (Supplemental Fig. 2) are consistent with this structure assignment. The aglycone ion at $m / z, 267$ is also formed via in-source fragmentation and showed the M19 product ions at $m / z$ 236, 208, and 162 upon $\mathrm{MS}^{2}$ fragmentation.

\section{M22}

The $[\mathrm{M}+\mathrm{H}]^{+}$of M22 at $\mathrm{m} / z 429$ was 176 Da higher than that of M20, pointing to glucuronidation of M20. This was confirmed by the elemental composition assignment and fragmentation providing the M20 MS ${ }^{2}$ product ions at $\mathrm{m} / \mathrm{z}, 236,225,208$, and 162 . The aglycone ion at $m / z, 253$ is also formed via in-source fragmentation and showed the diagnostic accurate mass product ions at $\mathrm{m} / \mathrm{z} 236,225,208$, and 162 upon $\mathrm{MS}^{2}$ selection.

\section{Discussion}

This open-label, phase 1 pharmacokinetic study was designed to assess the absorption, metabolic pathways, and route of excretion of orally administered apalutamide, with additional in vitro studies undertaken to assess the metabolism and phenotyping of apalutamide and M3 in human hepatocytes. Part A of this phase 1 study specifically determined the absolute bioavailability of apalutamide administered orally and via intravenous infusion using a microtracer approach for which microdose quantities of ${ }^{14} \mathrm{C}$-radiolabeled apalutamide were delivered. Administration of apalutamide was associated with a high absolute oral bioavailability of approximately 1.0 and low systemic clearance, consistent with that observed in single-dose in vivo pharmacokinetic studies in mouse and dog, which showed high oral bioavailability of approximately $93 \%$ (mouse) and $149 \%$ (dog) with a suspension or solution formulation, and low clearance of below $0.1 \mathrm{l} / \mathrm{h}$ per kg with a long plasma half-life of approximately 18 and 90 hours, respectively (Clegg et al., 2012).

Based on in vitro studies with Caco-2 cells, apalutamide was predicted to have high permeability in the human intestine; the apicalto-basolateral permeability was $42.3 \times 10^{-6} \mathrm{~cm} / \mathrm{s}$ (data on file). The high permeability and absolute oral bioavailability are further supported by the results from part B of the mass-balance study, where $88.9 \%$ of the orally administered apalutamide dose was recovered in urine $(64.6 \%)$ or in feces $(24.3 \%)$. The amount excreted in feces as unchanged apalutamide represents $1.54 \%$ of the administered dose, suggesting that 
A

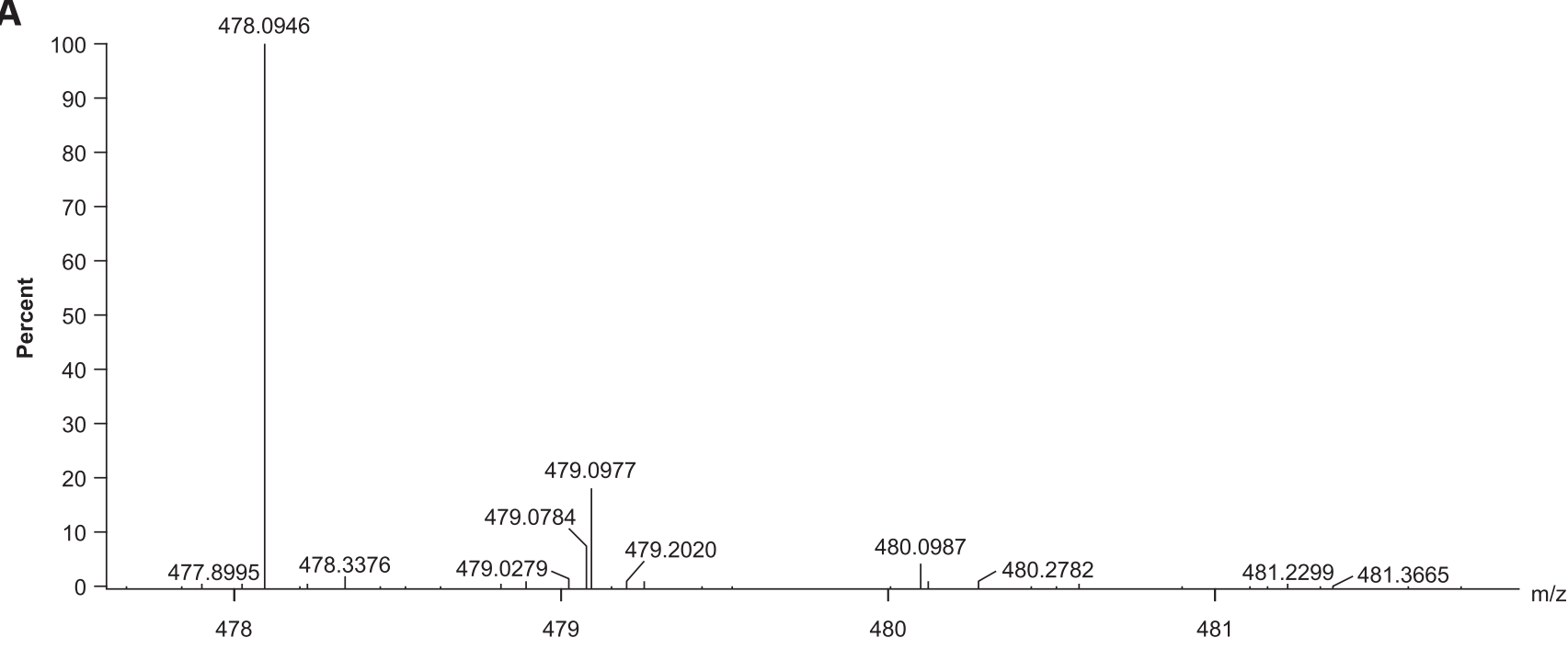

B

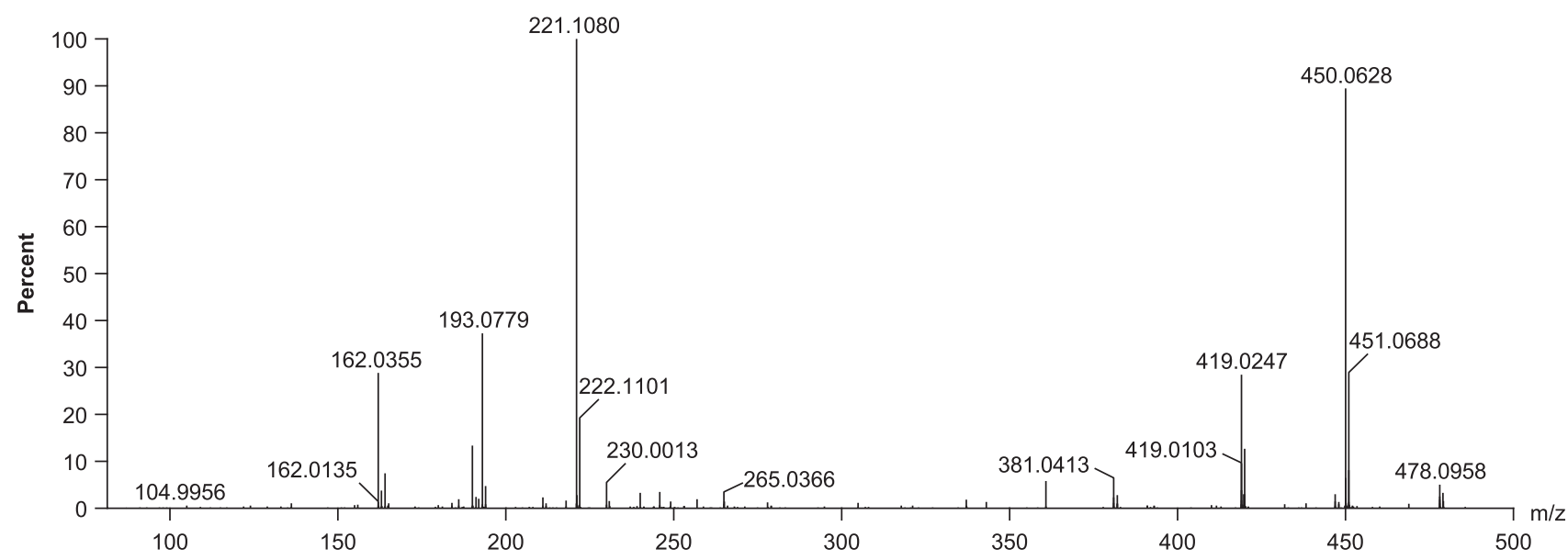

Fig. 6. Accurate mass full scan (A) and product ion mass spectra (B) of apalutamide.

the majority of the dose was absorbed after oral dosing. The AME profile of orally administered apalutamide was characterized by rapid initial absorption, as shown by a $\mathrm{T}_{\max }$ of $1.0-1.5$ hours followed by slow elimination over a period of several weeks, predominantly via urine in the form of metabolites, but also in feces to a smaller extent. Consistent with elimination kinetics, the metabolism of apalutamide to form its major metabolite $\mathrm{M} 3$ proceeded with a prolonged $\mathrm{T}_{\max }$ and elimination half-life of more than 1 week. M4, in contrast, had a $\mathrm{T}_{\max }$ of 3.0 hours, and 15-fold to 21-fold lower exposure compared with either apalutamide or M3. Based on systemic exposure, relative potency, and pharmacokinetic properties, M3 likely contributed to approximately $28 \%-32 \%$ of the clinical activity of apalutamide.

Six main metabolic clearance pathways for apalutamide could be distinguished based on the nature of the identified metabolites after a single dose of ${ }^{14} \mathrm{C}$-apalutamide. Of these, $\mathrm{N}$-demethylation of the $\mathrm{N}$-methylbenzamide moiety to M3 then benzamide hydrolysis to M4, together with direct benzamide hydrolysis to M4, are the most relevant clearance pathways. The relative importance of the two pathways on the formation of M4 is unknown. M4 is an inactive minor metabolite and is therefore of minor clinical relevance, but the formation of M4 is nevertheless important from a drug clearance perspective. Clearance of the parent drug as M3 without subsequent benzamide hydrolysis is minor, with only $4.5 \%$ of the dose excreted as M3. Metabolites of all pathways were common to both urinary and fecal elimination routes, with the exception of metabolites of the oxidation pathway, which were principally identified in urine alone.

There were no deaths or other serious AEs reported, and no discontinuations due to AEs. AEs that were considered possibly related to treatment were consistent with the tolerability profile reported for apalutamide in clinical studies (Rathkopf et al., 2013; Smith et al., 2016, 2018). Overall, single-dose treatment with $240 \mathrm{mg}$ of apalutamide plus a microdose ${ }^{14} \mathrm{C}$-labeled apalutamide was well tolerated in healthy men.

Because of the very low turnover of apalutamide and M3 in human liver S9 fractions and human liver microsomes, the effect of cytochrome P450 and esterase inhibitors was measured in human hepatocytes using itraconazole, gemfibrozil, and BNPP as specific inhibitors of CYP3A4, CYP2C8, and esterases, respectively. An in vitro relay method (Di et al., 2012) was used to investigate the metabolic pathways of hepatic clearance of apalutamide and the potential contribution of cytochrome P450 isoforms and esterases to the formation of M3 and M4. The addition of relay steps to prolong exposure time ensures that a drug such as apalutamide with a low intrinsic clearance can be continuously metabolized in vitro beyond the 4-hour limit of conventional human hepatocyte suspension incubations by regularly replacing cells with 
A

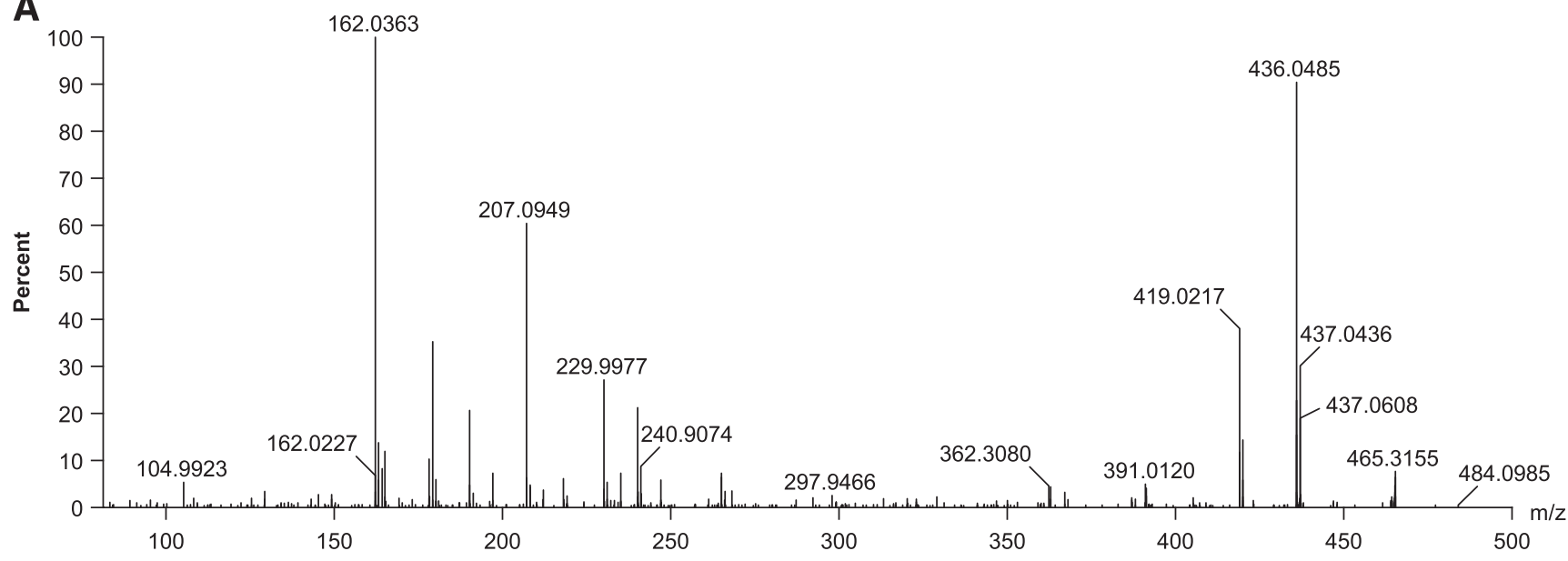

B

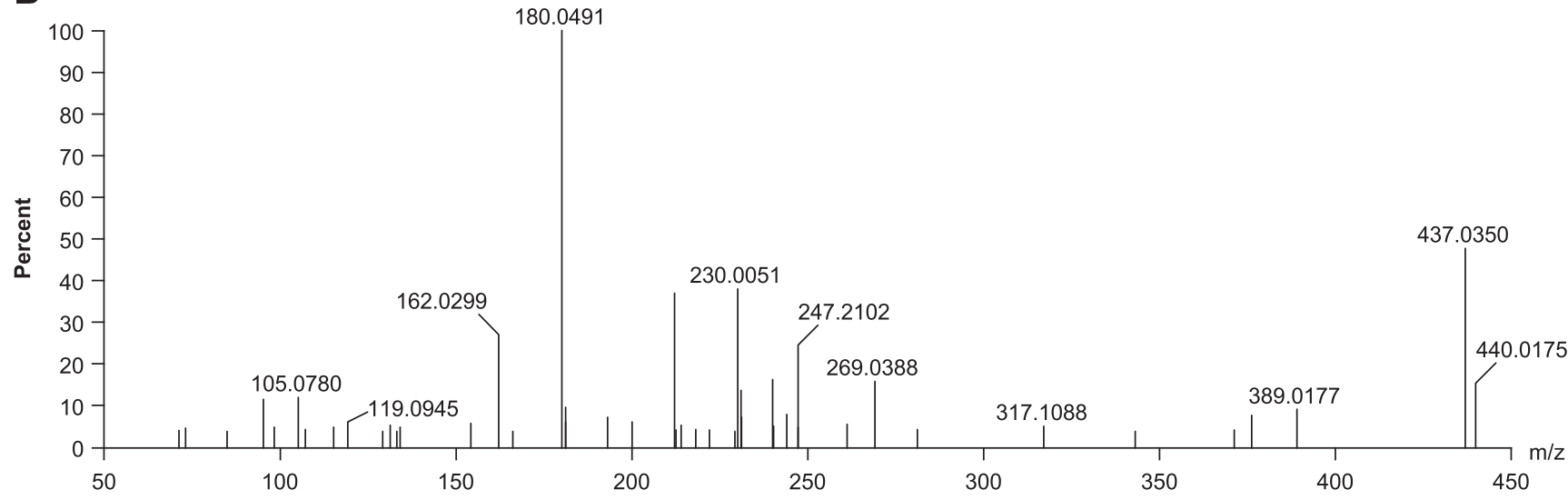

Fig. 7. Product ion mass spectra of M3 (A) and M4 (B).

freshly thawed hepatocytes. Diagnostic inhibitors of cytochrome P450 and esterase enzymes were used to demonstrate that the metabolism of apalutamide to M3 was predominantly mediated by CYP2C8 and, to a lesser extent, by CYP3A4. Various other drugs have been described in the literature that are dual substrates of CYP2C8 and CYP3A4 for $\mathrm{N}$-demethylation reactions. This is, for instance, the case for imatinib, tozasertib, and zopiclone (Backman et al., 2016). Although the inhibition of M3 formation was observed after incubation with the esterase inhibitor BNPP, this was possibly linked to the nonspecific inhibition of CYP2C8 and CYP3A4 by BNPP (Supplemental Table 1). BNPP completely inhibited the conversion of M3 to M4, whereas the effect of ABT on the formation of M4 was minimal, indicating that the observed inhibition with BNPP was not the result of nonspecific cytochrome P450 inhibition but rather was mediated predominantly by esterases, presumably carboxylesterases. Hydrolysis of a benzamide group, as for the biotransformation from M3 to M4, is a common biotransformation, and has been described for various other drugs, including ochratoxin A (Wu et al., 2011), dabigatran (Blech et al., 2008), GW695634 N-((4-(2-(4-chloro-2-(3-chloro-5-cyanobenzoyl) phenoxy)acetamido)-3-methylphenyl)sulfonyl)propanamide) (de Serres et al., 2010), and GSK1018921 (2-methoxy-N-(2-methyl-1-phenyl-2(pyrrolidin-1-yl)propyl)-4,6-bis(trifluoromethyl)benzamide) (Griffini et al., 2010).

This study had several limitations. The absolute bioavailability of apalutamide in part A was $111 \%$. This finding potentially reflects the variability of the two analytical methods applied (i.e., LC-MS/MS for the quantification of apalutamide and HPLC fractionation followed by AMS for quantification of ${ }^{14} \mathrm{C}$-apalutamide), with both methods having an accepted precision and accuracy within $15 \%$. There was no evidence of any relevant adsorption of the intravenous dose to the syringes or infusion lines, which could also explain the observed high absolute oral bioavailability of apalutamide. In part B, metabolism of apalutamide was evaluated after single-dose administration only. A previous pharmacokinetic study showed that apalutamide clearance increases over time as a result of autoinduction (ERLEADA, 2018), indicating that metabolism should ideally be evaluated at steady state after repeat dosing. Nonetheless, the metabolite profile based on the M3/apalutamide AUC ratio was comparable to that achieved with repeat dosing in the earlier study. However, the differential role of CYP2C8 and CYP3A4 in the metabolism of apalutamide is expected to change at steady state when CYP3A4 is fully induced.

In conclusion, this study showed that a single dose of apalutamide was completely absorbed with an absolute bioavailability of approximately $100 \%$ after oral administration. The long plasma half-life and low clearance of apalutamide, together with its observed pharmacokinetics, support once-daily oral administration. Apalutamide was extensively metabolized with little unchanged drug detected in the urine or feces, and with metabolic profiling confirming M3 as the major active metabolite. The low recovery of apalutamide and M3 in urine, with less than $4 \%$ of the dose excreted in urine as the parent compound and M3 combined, indicates that hepatic metabolism plays a major role in the overall elimination of apalutamide. In vitro findings confirmed the role of 
CYP2C8 and CYP3A4 in the metabolism of apalutamide, which was predominantly mediated by CYP2C8 and, to a lesser extent, by CYP3A4. The conversion of M3 to the inactive carboxylic acid metabolite M4 is mediated exclusively by esterases, likely carboxyesterases.

\section{Acknowledgments}

Editorial assistance was provided by William Turner, and Ira Mills, of PAREXEL, with funding from Janssen Global Services, LLC.

\section{Authorship Contributions}

Participated in research design: Snoeys and Chien.

Conducted experiments: de Vries and Jacobs.

Performed data analysis: de Vries, Jacobs, Snoeys, Cuyckens, and Chien.

Wrote or contributed to the writing of the manuscript: de Vries, Jacobs, Mannens, Snoeys, Cuyckens, Chien, and Ward.

\section{References}

Backman JT, Filppula AM, Niemi M, and Neuvonen PJ (2016) Role of cytochrome P450 2C8 in drug metabolism and interactions. Pharmacol Rev 68:168-241.

Berteotti A, Vacondio F, Lodola A, Bassi M, Silva C, Mor M, and Cavalli A (2014) Predicting the reactivity of nitrile-carrying compounds with cysteine: a combined computational and experimental study. ACS Med Chem Lett 5:501-505.

Blech S, Ebner T, Ludwig-Schwellinger E, Stangier J, and Roth W (2008) The metabolism and disposition of the oral direct thrombin inhibitor, dabigatran, in humans. Drug Metab Dispos 36: 386-399.

Clegg NJ, Wongvipat J, Joseph JD, Tran C, Ouk S, Dilhas A, Chen Y, Grillot K, Bischoff ED, Cai L, et al. (2012) ARN-509: a novel antiandrogen for prostate cancer treatment. Cancer Res 72:1494-1503.

de Serres M, Moss L, Sigafoos J, Sefler A, Castellino S, Bowers G, and Serabjit-Singh C (2010) The disposition and metabolism of GW695634: a non-nucleoside reverse transcriptase inhibitor (NNRTi) for treatment of HIV/AIDS. Xenobiotica 40:437-445.

Di L, Trapa P, Obach RS, Atkinson K, Bi YA, Wolford AC, Tan B, McDonald TS, Lai Y, and Tremaine LM (2012) A novel relay method for determining low-clearance values. Drug Metab Dispos 40:1860-1865.

ERLEADA. (2018) Package insert. Janssen Pharmaceutical Companies, Horsham, PA

Griffini P, James AD, Roberts AD, and Pellegatti M (2010) Metabolites in safety testing: issues and approaches to the safety evaluation of human metabolites in a drug that is extensively metabolized. J Drug Metab Toxicol 1:102.

Guerini E, Schadt S, Greig G, Haas R, Husser C, Zell M, Funk C, Hartung T, Gloge A, and Mallalieu NL (2017) A double-tracer technique to characterize absorption, distribution, metabolism and excretion (ADME) of $\left[{ }^{14} \mathrm{C}\right]$-basimglurant and absolute bioavailability after ora administration and concomitant intravenous microdose administration of $\left[{ }^{13} \mathrm{C}_{6}\right]$-labeled basimglurant in humans. Xenobiotica 47:144-153.
Hatfield MJ and Potter PM (2011) Carboxylesterase inhibitors. Expert Opin Ther Pat 21: $1159-1171$.

Helmer E, Nicolas JM, Long J, Roffel AF, Jones E, Chanteux H, Diaz N, Garratt H, and Bosje T (2017) A dual-administration microtracer technique to characterize the absorption, distribution, metabolism, and excretion of $\left[{ }^{14}\right.$ C]seletalisib (UCB5857) in healthy subjects. J Clin Pharmacol 57:1582-1590

Kalgutkar AS (2017) Liabilities associated with the formation of "hard" electrophiles in reactive metabolite trapping screens. Chem Res Toxicol 30:220-238.

Kassahun K, McIntosh I, Koeplinger K, Sun L, Talaty JE, Miller DL, Dixon R, Zajic S, and Stoch SA (2014) Disposition and metabolism of the cathepsin K inhibitor odanacatib in humans. Drug Metab Dispos 42:818-827.

Lappin G (2016) Approaches to intravenous clinical pharmacokinetics: recent developments with isotopic microtracers. J Clin Pharmacol 56:11-23.

Lin JH, Macomson B, Tunceli O, Pericone C, Behl AS, and Deshmukh S (2017) Association of prostate specific-antigen doubling time (PSADT) with metastasis-free survival (MFS) and overall survival (OS) in non-metastatic castration-resistant prostate cancer (nmCRPC) (Abstract). $J$ Clin Oncol 35 (15 Suppl):e16525.

Maltsev OV, Walter V, Brandl MJ, and Hintermann L (2013) Medium buffer effects on the condensation of L-cysteine and aryl nitriles to (R)-2-aryl-4,5-dihydrothiazole-4-carboxylic acids. Synthesis 45:2763-2767.

Morcos PN, Yu L, Bogman K, Sato M, Katsuki H, Kawashima K, Moore DJ, Whayman M, Nieforth K, Heinig K, et al. (2017) Absorption, distribution, metabolism and excretion (ADME) of the ALK inhibitor alectinib: results from an absolute bioavailability and mass balance study in healthy subjects. Xenobiotica 47:217-229.

Parkinson A, Kazmi F, Buckley DB, Yerino P, Ogilvie BW, and Paris BL (2010) Systemdependent outcomes during the evaluation of drug candidates as inhibitors of cytochrome P450 (CYP) and uridine diphosphate glucuronosyltransferase (UGT) enzymes: human hepatocytes versus liver microsomes versus recombinant enzymes. Drug Metab Pharmacokinet 25:16-27.

Rathkopf DE, Morris MJ, Fox JJ, Danila DC, Slovin SF, Hager JH, Rix PJ, Chow Maneval E, Chen I, Gönen M, et al. (2013) Phase I study of ARN-509, a novel antiandrogen, in the treatment of castration-resistant prostate cancer. J Clin Oncol 31:3525-3530.

Sevrioukova IF and Poulos TL (2013) Understanding the mechanism of cytochrome P450 3A4 recent advances and remaining problems. Dalton Trans 42:3116-3126.

Smith MR, Antonarakis ES, Ryan CJ, Berry WR, Shore ND, Liu G, Alumkal JJ, Higano CS, Chow Maneval E, Bandekar R, et al. (2016) Phase 2 study of the safety and antitumor activity of apalutamide (ARN-509), a potent androgen receptor antagonist, in the high-risk nonmetastatic castration-resistant prostate cancer cohort. Eur Urol 70:963-970.

Smith MR, Kabbinavar F, Saad F, Hussain A, Gittelman MC, Bilhartz DL, Wynne C, Murray R, Zinner NR, Schulman C, et al. (2005) Natural history of rising serum prostate-specific antigen in men with castrate nonmetastatic prostate cancer. J Clin Oncol 23:2918-2925.

Smith MR, Saad F, Chowdhury S, Oudard S, Hadaschik BA, Graff JN, Olmos D, Mainwaring PN Lee JY, Uemura H, et al.; SPARTAN Investigators (2018) Apalutamide treatment and metastasis-free survival in prostate cancer. $N$ Engl J Med 378:1408-1418.

Wu Q, Dohnal V, Huang L, Kuča K, Wang X, Chen G, and Yuan Z (2011) Metabolic pathways of ochratoxin A. Curr Drug Metab 12:1-10.

Address correspondence to: Ronald de Vries, Janssen Research \& Development, Turnhoutseweg 30, B-2340 Beerse, Belgium. E-mail: rdvries@its.jnj.com 